JOURNAL OF

SYMPLECTIC GEOMETRY

Volume 1 Number 4, 715-765, 2002

\title{
FLOER HOMOLOGY OF ALGEBRAICALLY FINITE MAPPING CLASSES
}

\section{Ralf Gautschi}

\begin{abstract}
Using symplectic Floer homology, Seidel associated a module to each mapping class of a compact connected oriented two-manifold of genus bigger than one. We compute this module for mapping classes which do not have any pseudo-Anosov components in the sense of Thurston's theory of surface diffeomorphisms. The Nielsen-Thurston representative of such a class is shown to be monotone. The formula for the Floer homology is obtained from a topological separation of fixed points and a separation mechanism for Floer connecting orbits. As examples, we consider the geometric monodromy of isolated plane curve singularities. In this case, the formula for the Floer homology is particularly simple.
\end{abstract}

\section{Introduction}

This work is concerned with the computation of Floer homology groups of symplectomorphisms of compact 2-manifolds. In the case of a 2sphere, each symplectomorphism is exact and its Floer homology equals, by Floer's original work [11], the singular homology of the 2-sphere. In the case of a torus, the Floer homology of linear symplectomorphisms was computed by Poźniak [25].

In this article, we consider the case of a compact connected oriented 2-manifold $\Sigma$ of genus $\geq 2$. It was shown by Seidel [28] that to every mapping class $g$ of $\Sigma$, there is associated a $\mathbb{Z}_{2}$-graded vector space 
$H F_{*}(g)$ over the field $\mathbb{Z}_{2}$, which is equipped with an additional module structure over $H^{*}\left(\Sigma ; \mathbb{Z}_{2}\right)$. To put it short, $H F_{*}(g)$ is the symplectic Floer homology of an area preserving and so-called monotone representative of $g$.

The first computational result in this context is also due to Seidel. In [26] it was shown that if $g$ is the mapping class of a positive Dehn twist along an embedded circle $C \subset \Sigma$, then $H F_{*}(g) \cong H_{*}\left(\Sigma, C ; \mathbb{Z}_{2}\right)$.

Our starting point is the following definition given in detail in Section 3: an orientation preserving diffeomorphism $\phi$ of $\Sigma$ is called of finite type if $\Sigma$ can be obtained by piecing together $\phi$-invariant 2 -manifolds $\Sigma^{\prime}$ with boundary such that $\phi \mid \Sigma^{\prime}$ is either periodic, a flip-twist map or a twist map without fixed points. For the terminology we refer to Section 3. The significance of this definition lies in the following fact: due to Nielsen-Thurston theory of surface diffeomorphisms, every mapping class without pseudo-Anosov components admits a representative which is of finite type. Such a mapping class is called algebraically finite.

Our main result is a formula for the Floer homology of finite type diffeomorphisms. We use the following notation. If $\phi$ is such a diffeomorphism, we denote by $\Sigma_{0}$ the union of the $\Sigma^{\prime}$ where $\phi$ restricts to the identity. By $\partial_{+} \Sigma_{0}$, we denote the union of components of $\partial \Sigma_{0}$ where in a neighborhood, $\phi$ is a right-handed twist.

Theorem 1. If $\phi$ is a diffeomorphism of finite type, then $\phi$ is monotone with respect to some $\phi$-invariant area form and

$$
H F_{*}(\phi) \cong H_{*}\left(\Sigma_{0}, \partial_{+} \Sigma_{0} ; \mathbb{Z}_{2}\right) \oplus \mathbb{Z}_{2}^{\Lambda\left(\phi|\Sigma| \Sigma_{0}\right)} .
$$

Here, $\Lambda$ denotes the Lefschetz number. The $H^{*}\left(\Sigma ; \mathbb{Z}_{2}\right)$-action on the r.h.s. is split and given as follows. On the first summand, it is the ordinary cap product. On the second, $1 \in H^{0}\left(\Sigma ; \mathbb{Z}_{2}\right)$ acts by the identity and any element of $H^{1}\left(\Sigma ; \mathbb{Z}_{2}\right) \oplus H^{2}\left(\Sigma ; \mathbb{Z}_{2}\right)$ by the zero map.

As suggested by the formula above, the Floer complex of $\phi$ splits into a complex associated to $\phi \mid \Sigma_{0}$ and one associated to $\phi \mid \Sigma \backslash \Sigma_{0}$. On one hand this follows from a purely topological separation of fixed points for finite type diffeomorphisms. On the other hand, there is also a separation mechanism for Floer connecting orbits, i.e. after suitably perturbing $\phi$, every connecting orbit starting and ending in $\Sigma_{0}$ does not leave $\Sigma_{0}$. For the precise statement we refer to Section 4 .

A natural source of examples is provided by the theory of singularities. To every isolated plane curve singularity is associated a compact 
connected oriented 2-manifold with boundary, the Milnor fiber, and an isotopy class of orientation preserving diffeomorphisms of the Milnor fiber which are the identity near the boundary, called the geometric monodromy. The precise definitions are given in Section 6 .

Theorem 2. Let $M \subset \Sigma$ be the Milnor fiber of an isolated plane curve singularity and $g$ be the mapping class which is obtained by extending the geometric monodromy trivially to $\Sigma$. Then

$$
H F_{*}(g) \cong H_{*}\left(\Sigma, M ; \mathbb{Z}_{2}\right),
$$

where $H^{*}\left(\Sigma ; \mathbb{Z}_{2}\right)$ acts by cap product.

A special case of Theorem 2 is the following generalization of Seidel's formula for the Floer homology of a Dehn twist [26]. For the notation see Section 6.

Corollary 3. Let $\left(C_{1}, \ldots, C_{k}\right)$ be an $A_{k}$-configuration of circles in $\Sigma$. Let $g$ be the mapping class of the product $\tau_{1} \circ \cdots \circ \tau_{k}$, where $\tau_{i}$ denotes the positive Dehn twist along $C_{i}$. Then

$$
H F_{*}(g) \cong H_{*}\left(\Sigma, C_{1} \cup \cdots \cup C_{k} ; \mathbb{Z}_{2}\right),
$$

where $H^{*}\left(\Sigma ; \mathbb{Z}_{2}\right)$ acts by cap product. The same formula holds, if $g$ is the class of $\tau_{\sigma 1} \circ \cdots \circ \tau_{\sigma k}$, where $\sigma$ is a cyclic permutation of $k$ elements.

The proof of Theorem 2 is given in Section 6. It relies, besides on Theorem 1, on an additional result about the geometric monodromy. This result is stated in Proposition 19 and proven in Appendix B. The proof uses the well known theorem of A'Campo [2] and Lê [17] that the Lefschetz number of the geometric monodromy vanishes, together with the theory of splice diagrams which is due to Eisenbud and Neumann [10]. Appendix B includes a summary of the relevant results on splice diagrams from [10] and is therefore quite lengthy. We would like to point out that Proposition 19 also follows from A'Campo's work [1], $[4]$.

Finally, we mention that there is a version of Floer homology theory for diffeomorphisms of compact oriented 2-manifolds with boundary. It assigns a pair of vector spaces $H F_{*}(g,+), H F_{*}(g,-)$ to every isotopy class $g$ of orientation preserving diffeomorphisms which are the identity near the boundary. The sign corresponds to two different perturbations of $g$ near the boundary. In Appendix $\mathrm{C}$ we give an outline on this version of Floer homology. Using Proposition 19, we confirm in Section 6 a conjecture of Seidel [27, Page 23] in the case of plane curve singularities. 
Theorem 4. If $g$ is the geometric monodromy of an isolated plane curve singularity, then

$$
H F_{*}(g,+)=0 .
$$

The rest of the article is organized as follows. In Section 2 we recall the basic facts about monotone symplectomorphisms and Floer homology. For background material on symplectic Floer homology in two dimensions we refer to [28] and the references given therein. Section 3 is devoted to diffeomorphisms of finite type and their properties relevant for Floer homology. We compute the fixed point classes, establish monotonicity and show that the symplectic action is exact. At several points we use a topological proposition about products of disjoint Dehn twists which was already used in [26]. We give a proof of this proposition in Appendix A. The result about the fixed point classes, Proposition 9, is already contained in the work of Jiang and Guo [16] on the Nielsen number of surface diffeomorphisms. For the sake of completeness, we give an independent proof. In Section 5, the results from the previous sections are put together to prove Theorem 1.

We would like to point out that the proof of Theorem 1 is very much inspired by Seidel's computation of the Floer homology of a Dehn twist [26]. In particular, our result about the Floer connecting orbits, Proposition 14, is a generalization of the corresponding result for Dehn twists [26, Lemma 4]. Our approach to the proof, however, is slightly different from Seidel's original approach. This has led to a shorter proof and to a wider range of application, see the remark at the end of Section 4 .

At this point we would like to mention two recent articles on the computation of Floer homology groups in two dimensions. The first is Eftekhary's [9] computation of the Floer homology of certain products of Dehn twists which represent pseudo-Anosov mapping classes. In the second, Hutchings and Sullivan [14] compute the so-called periodic Floer homology, a generalization of symplectic Floer homology, for the Dehn twist.

ACKNOWLEDGEMEnT. The idea of looking at algebraically finite mapping classes was the fruit of a week of stimulating discussions with Paul Seidel at Ecole polytechnique. I am indebted to him for devoting time and sharing his insight. This is a part of my PhD thesis and I am very grateful to my supervisor Dietmar Salamon for all his advise during the preparation of this work. I thank Eduard Zehnder for many helpful suggestions. 


\section{Monotonicity and Floer homology}

In this section we discuss the notion of monotonicity as defined in [28] and give an outline of its significance for Floer homology in two dimensions. For a more detailed account we refer to the original article. At the end of this section we give two criteria for monotonicity which we use in the next section. Throughout this article, $\Sigma$ denotes a closed connected and oriented 2-manifold of genus $\geq 2$. In this section, we also fix an area form $\omega$ on $\Sigma$.

Let $\phi \in \operatorname{Symp}(\Sigma, \omega)$, the group of $\omega$-preserving diffeomorphisms of $\Sigma$. The mapping torus of $\phi$,

$$
T_{\phi}=\mathbb{R} \times \Sigma /(t+1, x) \sim(t, \phi(x)),
$$

is a 3-manifold fibered over $S^{1}=\mathbb{R} / \mathbb{Z}$. There are two natural second cohomology classes on $T_{\phi}$, denoted by $\left[\omega_{\phi}\right]$ and $c_{\phi}$. The first one is represented by the closed two-form $\omega_{\phi}$ which is induced from the pullback of $\omega$ to $\mathbb{R} \times \Sigma$. The second is the Euler class of the vector bundle

$$
V_{\phi}=\mathbb{R} \times T \Sigma /\left(t+1, \xi_{x}\right) \sim\left(t, \mathrm{~d} \phi_{x} \xi_{x}\right),
$$

which is of rank 2 and inherits an orientation from $T \Sigma$.

Definition 5 (Seidel). $\phi \in \operatorname{Symp}(\Sigma, \omega)$ is called monotone if

$$
\left[\omega_{\phi}\right]=\left(\operatorname{area}_{\omega}(\Sigma) / \chi(\Sigma)\right) \cdot c_{\phi}
$$

in $H^{2}\left(T_{\phi} ; \mathbb{R}\right) ; \operatorname{Symp}^{m}(\Sigma, \omega)$ denotes the set of monotone symplectomorphisms.

Now $H^{2}\left(T_{\phi} ; \mathbb{R}\right)$ fits into the following short exact sequence

$$
0 \longrightarrow \frac{H^{1}(\Sigma ; \mathbb{R})}{\mathrm{im}\left(\mathrm{id}-\phi^{*}\right)} \stackrel{\delta}{\longrightarrow} H^{2}\left(T_{\phi} ; \mathbb{R}\right) \stackrel{\iota^{*}}{\longrightarrow} H^{2}(\Sigma ; \mathbb{R}) \longrightarrow 0 .
$$

The map $\delta$ is defined as follows. Let $\rho:[0,1] \rightarrow \mathbb{R}$ be a smooth function which vanishes near 0 and 1 and satisfies $\int_{0}^{1} \rho \mathrm{d} t=1$. If $\theta$ is a closed 1-form on $\Sigma$, then $\rho \cdot \theta \wedge \mathrm{d} t$ defines a closed 2-form on $T_{\phi}$; indeed

$$
\delta[\theta]=[\rho \cdot \theta \wedge \mathrm{d} t] .
$$

The map $\iota: \Sigma \hookrightarrow T_{\phi}$ assigns to each $x \in \Sigma$ the equivalence class of $(1 / 2, x)$. Note that $\iota^{*} \omega_{\phi}=\omega$ and $\iota^{*} c_{\phi}$ is the Euler class of $T \Sigma$. Hence, by (1), there exists a unique class $m(\phi) \in H^{1}(\Sigma ; \mathbb{R}) / \mathrm{im}\left(\mathrm{id}-\phi^{*}\right)$ satisfying

$$
\delta m(\phi)=\left[\omega_{\phi}\right]-\left(\operatorname{area}_{\omega}(\Sigma) / \chi(\Sigma)\right) \cdot c_{\phi},
$$

where $\chi$ denotes the Euler characteristic. Therefore, $\phi$ is monotone if and only if $m(\phi)=0$. 
We recall the fundamental properties of $\operatorname{Symp}^{m}(\Sigma, \omega)$ which are discussed in [28]. By $\operatorname{Diff}^{+}(\Sigma)$, we denote the group of orientation preserving diffeomorphisms of $\Sigma$.

(Naturality) If $\phi \in \operatorname{Symp}^{m}(\Sigma, \omega)$, then $\psi^{-1} \phi \psi \in \operatorname{Symp}^{m}\left(\Sigma, \psi^{*} \omega\right)$ for all $\psi \in \operatorname{Diff}^{+}(\Sigma)$.

(Isotopy) Let $\left(\psi_{t}\right)_{t \in[0,1]}$ be an isotopy in $\operatorname{Symp}(\Sigma, \omega)$, i.e. a smooth path with $\psi_{0}=$ id. Then

$$
m\left(\phi \circ \psi_{1}\right)=m(\phi)+\left[\operatorname{Flux}\left(\psi_{t}\right)_{t \in[0,1]}\right]
$$

in $H^{1}(\Sigma ; \mathbb{R}) / \operatorname{im}\left(\mathrm{id}-\phi^{*}\right)$; see $[\mathbf{2 8}$, Lemma 6$]$. For the definition of the flux homomorphism see $[\mathbf{1 9}]$.

(Inclusion) The inclusion $\operatorname{Symp}^{m}(\Sigma, \omega) \hookrightarrow \operatorname{Diff}^{+}(\Sigma)$ is a homotopy equivalence. This follows from the isotopy property, surjectivity of the flux homomorphism and Moser's isotopy theorem [20]. Furthermore, the Earl-Eells Theorem [8] implies that every connected component of $\operatorname{Symp}^{m}(\Sigma, \omega)$ is contractible.

(Floer homology) To every $\phi \in \operatorname{Symp}^{m}(\Sigma, \omega)$ symplectic Floer homology theory assigns a $\mathbb{Z}_{2}$-graded vector space $H F_{*}(\phi)$ over $\mathbb{Z}_{2}$, with an additional multiplicative structure, called the quantum cap product,

$$
H^{*}\left(\Sigma ; \mathbb{Z}_{2}\right) \otimes H F_{*}(\phi) \longrightarrow H F_{*}(\phi) .
$$

Each $\psi \in \operatorname{Diff}^{+}(\Sigma)$ induces an isomorphism $H F_{*}(\phi) \cong H F_{*}\left(\psi^{-1} \phi \psi\right)$ of $H^{*}\left(\Sigma ; \mathbb{Z}_{2}\right)$-modules.

(Invariance) If $\phi$ and $\phi^{\prime} \in \operatorname{Symp}^{m}(\Sigma, \omega)$ are isotopic in $\operatorname{Diff}^{+}(\Sigma)$, then $H F_{*}(\phi)$ and $H F_{*}\left(\phi^{\prime}\right)$ are naturally isomorphic as $H^{*}\left(\Sigma ; \mathbb{Z}_{2}\right)$-modules. This is proven in [28, Page 7]. Note that by the inclusion property, $\phi$ and $\phi^{\prime}$ are isotopic in $\operatorname{Symp}^{m}(\Sigma, \omega)$ if and only if they are isotopic in $\operatorname{Diff}^{+}(\Sigma)$.

Now let $g$ be a mapping class of $\Sigma$, i.e. an isotopy class of $\operatorname{Diff}^{+}(\Sigma)$. Pick an area form $\omega$ and a representative $\phi \in \operatorname{Symp}^{m}(\Sigma, \omega)$ of $g$. Then $H F_{*}(\phi)$ is an invariant of $g$, which is denoted by $H F_{*}(g)$. Note that $H F_{*}(g)$ is independent of the choice of an area form $\omega$ by Moser's isotopy theorem [20] and naturality of Floer homology.

Let $\phi \in \operatorname{Symp}^{m}(\Sigma, \omega)$. We give a brief outline of the definition of $H F_{*}(\phi)$ in the special case where all the fixed points of $\phi$ are nondegenerate. This means that for all $y \in \operatorname{Fix}(\phi), \operatorname{det}\left(\mathrm{id}-\mathrm{d} \phi_{y}\right) \neq 0$. In particular, it follows that $\operatorname{Fix}(\phi)$ is a finite set and the $\mathbb{Z}_{2}$-vector space

$$
C F_{*}(\phi):=\mathbb{Z}_{2}^{\operatorname{Fix}(\phi)}
$$


admits a $\mathbb{Z}_{2}$-grading with $(-1)^{\operatorname{deg} y}=\operatorname{sign}\left(\operatorname{det}\left(\mathrm{id}-\mathrm{d} \phi_{y}\right)\right)$, for all $y \in$ $\operatorname{Fix}(\phi)$. The Floer boundary operator is defined as follows. Let $J=$ $\left(J_{t}\right)_{t \in \mathbb{R}}$ be a smooth path of $\omega$-compatible complex structures on $\Sigma$ such that $J_{t+1}=\phi^{*} J_{t}$. For $y^{ \pm} \in \operatorname{Fix}(\phi)$, let $\mathcal{M}\left(y^{-}, y^{+} ; J, \phi\right)$ denote the space of smooth maps $u: \mathbb{R}^{2} \rightarrow \Sigma$ which satisfy the Floer equations

$$
\left\{\begin{array}{l}
u(s, t)=\phi(u(s, t+1)), \\
\partial_{s} u+J_{t}(u) \partial_{t} u=0 \\
\lim _{s \rightarrow \pm \infty} u(s, t)=y^{ \pm} .
\end{array}\right.
$$

One way to think of the Floer equations is in terms of the symplectic action. Let $\Omega_{\phi}=\left\{y \in C^{\infty}(\mathbb{R}, \Sigma) \mid y(t)=\phi(y(t+1))\right\}$ denote the twisted loop space. The action form is the one-form $\alpha_{\omega}$ on $\Omega_{\phi}$ defined by

$$
\alpha_{\omega}(y) \xi=\int_{0}^{1} \omega\left(\frac{\mathrm{d} y}{\mathrm{~d} t}(t), \xi(t)\right) \mathrm{d} t
$$

where $y \in \Omega_{\phi}$ and $\xi \in T_{y} \Omega_{\phi}$, i.e. $\xi(t) \in T_{y(t)} \Sigma$ and $\xi(t)=\mathrm{d} \phi_{y(t+1)} \xi(t+1)$ for all $t \in \mathbb{R}$. If $\xi, \xi^{\prime} \in T_{y} \Omega_{\phi}$, then $\int_{0}^{1} \omega\left(\xi^{\prime}(t), J_{t} \xi(t)\right) \mathrm{d} t$ defines a metric on $\Omega_{\phi}$. The negative gradient lines of $\alpha_{\omega}$ with respect to this metric are solutions of (2).

Now to every $u \in \mathcal{M}\left(y^{-}, y^{+} ; J, \phi\right)$ is associated a Fredholm operator $\mathrm{D}_{u}$ which linearizes (2) in suitable Sobolev spaces. The index of this operator is given by the so called Maslov index $\mu(u)$, which satisfies $\mu(u)=$ $\operatorname{deg}\left(y^{+}\right)-\operatorname{deg}\left(y^{-}\right) \bmod 2$. For a generic $J$, every $u \in \mathcal{M}\left(y^{-}, y^{+} ; J, \phi\right)$ is regular, meaning that $\mathrm{D}_{u}$ is onto. Hence, by the implicit function theorem, $\mathcal{M}_{k}\left(y^{-}, y^{+} ; J, \phi\right)$ is a smooth $k$-dimensional manifold. Here $\mathcal{M}_{k}\left(y^{-}, y^{+} ; J, \phi\right)$ denotes the subset of those $u \in \mathcal{M}\left(y^{-}, y^{+} ; J, \phi\right)$ with $\mu(u)=k \in \mathbb{Z}$.

Translation of the $s$-variable defines a free $\mathbb{R}$-action on $\mathcal{M}_{1}\left(y^{-}, y^{+} ; J, \phi\right)$ and hence the quotient is a discrete set of points. Assume for the moment that for all $y^{ \pm} \in \operatorname{Fix}(\phi)$ this quotient is a finite set and let $n\left(y^{-}, y^{+}\right) \in \mathbb{Z}_{2}$ denote its cardinality $\bmod 2$. Define the linear map

$$
\partial_{J}: C F_{*}(\phi) \longrightarrow C F_{*+1}(\phi) \quad \text { by } \quad \operatorname{Fix}(\phi) \ni y \longmapsto \sum_{z} n(y, z) z .
$$

That $\partial_{J}$ is of degree 1 follows from the equation relating the index and the degree. That $\partial_{J}$ is a boundary operator, i.e. that $\partial_{J} \circ \partial_{J}=0$, is due to the so-called gluing theorem. For this theorem to hold, as well as for $\mathcal{M}_{1}\left(y^{-}, y^{+} ; J, \phi\right) / \mathbb{R}$ to be a finite set, one needs certain bounds on the energy. Note that bubbling is not an issue here, since $\pi_{2}(\Sigma)=0$. The 
energy of a map $u: \mathbb{R}^{2} \rightarrow \Sigma$ is given by

$$
E(u)=\int_{\mathbb{R}} \int_{0}^{1} \omega\left(\partial_{t} u(s, t), J_{t} \partial_{t} u(s, t)\right) \mathrm{d} t \mathrm{~d} s .
$$

It is proven in [28, Lemma 9] that if $\phi$ is monotone, then the energy is constant on each $\mathcal{M}_{k}\left(y^{-}, y^{+} ; J, \phi\right)$. It follows that $\left(C F_{*}(\phi), \partial_{J}\right)$ is a chain complex and that its homology is an invariant of $\phi$, denoted by $H F_{*}(\phi)$, i.e. it is independent of $J$.

Next we introduce the quantum cap product on $H F_{*}(\phi)$. For this, choose a Morse function $f: \Sigma \rightarrow \mathbb{R}$ and set

$$
C M^{*}(f):=\mathbb{Z}_{2}^{\operatorname{Crit}(f)},
$$

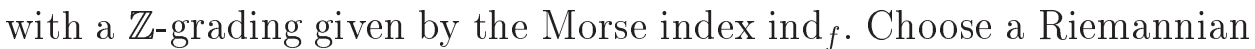
metric on $\Sigma$ such that $\nabla f$ is a Morse-Smale vector field. If $x^{ \pm} \in \operatorname{Crit}(f)$ and $\operatorname{ind}_{f}\left(x^{+}\right)=\operatorname{ind}_{f}\left(x^{-}\right)+1$, denote by $l\left(x^{-}, x^{+}\right) \in \mathbb{Z}_{2}$ the number mod 2 of positive gradient lines going from $x^{-}$to $x^{+}$. Define the Morse coboundary operator

$$
\delta_{\nabla f}: C M^{*}(f) \longrightarrow C M^{*+1}(f) \quad \text { by } \quad \operatorname{Crit}(f) \ni x \longmapsto \sum_{y} l(x, y) y .
$$

The cohomology of $\left(C M^{*}(f), \delta_{\nabla f}\right)$ is naturally isomorphic to $H^{*}\left(\Sigma ; \mathbb{Z}_{2}\right)$, see [24]. Now by a suitable choice of the function $f$ or the metric, we may assume that for all $y^{ \pm} \in \operatorname{Fix}(\phi), x \in \operatorname{Crit}(f)$ and $k \in \mathbb{Z}$, the evaluation map

$$
\eta_{k}: \mathcal{M}_{k}\left(y^{-}, y^{+} ; J, \phi\right) \longrightarrow \Sigma, \quad u \longmapsto u(0,0),
$$

is transverse to the unstable manifold $W^{u}(\nabla f, x) \subset \Sigma$. Note that the dimension of $W^{u}(\nabla f, x)$ is $2-\operatorname{ind}_{f}(x)$. Hence if $k=\operatorname{ind}_{f}(x)$, then $\eta_{k}^{-1}\left(W^{u}(f, x)\right)$ is a discrete set of points. It is in fact a finite set, see [28, page 8]. To prove this one uses the Gromov-Floer compactification of the moduli spaces and the fact that $\pi_{2}(\Sigma)=0$. Denote by $q\left(x ; y^{-}, y^{+}\right) \in$ $\mathbb{Z}_{2}$ the cardinality mod 2 of $\eta_{k}^{-1}\left(W^{u}(f, x)\right) \subset \mathcal{M}_{k}\left(y^{-}, y^{+} ; J, \phi\right)$, where $k=\operatorname{ind}_{f}(x)$. Define the linear map

$$
C M^{*}(f) \otimes C F_{*}(\phi) \longrightarrow C F_{*}(\phi), \quad x \otimes y \longmapsto \sum_{z} q(x ; y, z) z .
$$

It can be shown that this is a chain map and that the induced map on homology is independent of $\nabla f$ and $J$. It is called the quantum cap product. For details we refer to [28] and the references given therein.

If $\phi$ has degenerate fixed points one needs to perturb equations (2) in order to define the Floer homology. Equivalently, one could say that 
the action form needs to be perturbed. At this point Seidel's approach differs from the usual one. He uses a larger class of perturbations, but such that the perturbed action form is still cohomologous to the unperturbed. As a consequence, the usual invariance of Floer homology under Hamiltonian isotopies is extended to the stronger property stated above. This ends the general discussion of Floer homology .

To compute the Floer homology of a mapping class one needs to pick a monotone representative. We now give two criteria for monotonicity which we use later on. Let $\omega$ be an area form on $\Sigma$ and $\phi \in \operatorname{Symp}(\Sigma, \omega)$.

Lemma 6. Assume that every class $\alpha \in \operatorname{ker}\left(\mathrm{id}-\phi_{*}\right) \subset H_{1}(\Sigma ; \mathbb{Z})$ is represented by a map $\gamma: S \rightarrow \operatorname{Fix}(\phi)$, where $S$ is a compact oriented 1-manifold. Then $\phi$ is monotone.

Proof. By dualizing the exact sequence (1), we get the following exact sequence for homology with real coefficients

$$
0 \longrightarrow H_{2}(\Sigma ; \mathbb{R}) \stackrel{\iota_{*}}{\longrightarrow} H_{2}\left(T_{\phi} ; \mathbb{R}\right) \stackrel{\hat{\partial}}{\longrightarrow} \operatorname{ker}\left(\mathrm{id}-\phi_{*}\right) \subset H_{1}(\Sigma ; \mathbb{R}),
$$

where $\hat{\partial}$ is dual to $\delta$. Hence, $\phi$ is monotone if and only if

$$
\langle m(\phi), \alpha\rangle=0, \quad \forall \alpha \in \operatorname{ker}\left(\mathrm{id}-\phi_{*}\right) \subset H_{1}(\Sigma ; \mathbb{R}) .
$$

If we think of $H_{1}(\Sigma ; \mathbb{Z})$ as a lattice in $H_{1}(\Sigma ; \mathbb{R})$, it is furthermore enough to consider $\alpha \in H_{1}(\Sigma ; \mathbb{Z}) \cap \operatorname{ker}\left(\mathrm{id}-\phi_{*}\right)$.

Let $\gamma: S \rightarrow \operatorname{Fix}(\phi)$ and define $u: S \times S^{1} \rightarrow T_{\phi}$ by $u(s, t)=(t, \gamma(s))$. From the definition of $\delta$ on page 719 , it is straight forward to check that

$$
\langle\delta \beta,[u]\rangle=\langle\beta,[\gamma]\rangle
$$

for all $\beta \in H_{1}(\Sigma ; \mathbb{R})$, i.e. that $\hat{\partial}[u]=[\gamma]$. Here, the brackets denote homology classes. Now on one hand, since $\partial_{t} u(s, t)=(1,0)$, we have that $u^{*} \omega_{\phi}=0$ and hence $\left\langle\left[\omega_{\phi}\right],[u]\right\rangle=0$. On the other hand, $\left\langle c_{\phi},[u]\right\rangle=$ 0 . This is because the bundle $u^{*} V_{\phi}$ is isomorphic to the bundle $\gamma^{*} T \Sigma \times$ $S^{1}$, which is trivial. Hence, it follows that

$$
\langle m(\phi),[\gamma]\rangle=\left\langle\left[\omega_{\phi}\right],[u]\right\rangle-\left(\operatorname{area}_{\omega}(\Sigma) / \chi(\Sigma)\right)\left\langle c_{\phi},[u]\right\rangle=0 .
$$

This proves the lemma.

Lemma 7. If $\phi^{k}$ is monotone for some $k>0$, then $\phi$ is monotone. If $\phi$ is monotone, then $\phi^{k}$ is monotone for all $k>0$.

Proof. Recall that $T_{\phi}$ is the orbit space of the $\mathbb{Z}$-action $n \cdot(t, x)=$ $\left(t+n, \phi^{-n}(x)\right)$, where $n \in \mathbb{Z}$ and $(t, x) \in \mathbb{R} \times \Sigma$. If we only divide out by the subgroup $k \mathbb{Z}$, for $k \in \mathbb{N}_{>0}$, we naturally get the mapping 
torus of $\phi^{k}$. Further dividing by $\mathbb{Z} / k \mathbb{Z}$ defines the $k$-fold covering map $p_{k}: T_{\phi^{k}} \rightarrow T_{\phi}$. It is straight forward to check that

$$
p_{k}^{*}\left[\omega_{\phi}\right]=\left[\omega_{\phi^{k}}\right] \text { and } p_{k}^{*} c_{\phi}=c_{\phi^{k}} .
$$

The first equality follows immediately from the definitions. To prove the second, note that

$$
p_{k}^{*}((T M \times \mathbb{R}) / \mathbb{Z}) \cong(T M \times \mathbb{R}) / k \mathbb{Z} \cong V_{\phi^{k}},
$$

where the $\mathbb{Z}$-action on $\mathbb{R} \times T \Sigma$ is given by $n \cdot\left(t, \xi_{x}\right)=\left(t+n, \mathrm{~d} \phi_{x}^{-n} \xi_{x}\right)$, for $n \in \mathbb{Z}$ and $\xi_{x} \in T_{x} \Sigma$. The lemma follows from (6) and the fact that $p_{k}^{*}$ is injective. To prove injectivity, define the map $a_{*}^{k}: H^{2}\left(T_{\phi^{k}} ; \mathbb{R}\right) \rightarrow$ $H^{2}\left(T_{\phi} ; \mathbb{R}\right)$ by averaging differential forms; $a_{*}^{k}$ is a left inverse of $p_{k}^{*}$, i.e. $a_{*}^{k} \circ p_{k}^{*}=\mathrm{id}$. This ends the proof of the lemma.

\section{Diffeomorphisms of finite type}

We begin with the basic definition. Note that $S^{1}$ is always identified with $\mathbb{R} / \mathbb{Z}$.

Definition 8. We call $\phi \in \operatorname{Diff}_{+}(\Sigma)$ of finite type if the following holds. There is a $\phi$-invariant finite union $N \subset \Sigma$ of disjoint noncontractible annuli such that:

(1) $\phi \mid \Sigma \backslash N$ is periodic, i.e. there exists $\ell>0$ such that $\phi^{\ell} \mid \Sigma \backslash N=\mathrm{id}$.

(2) Let $N^{\prime}$ be a connected component of $N$ and $\ell^{\prime}>0$ be the smallest integer such that $\phi^{\ell^{\prime}}$ maps $N^{\prime}$ to itself. Then $\phi^{\ell^{\prime}} \mid N^{\prime}$ is given by one of the following two models with respect to some coordinates $(q, p) \in[0,1] \times S^{1}:$

(twist map)

$$
\begin{aligned}
& (q, p) \longmapsto(q, p-f(q)) \\
& (q, p) \longmapsto(1-q,-p-f(q)),
\end{aligned}
$$

(flip-twist map)

where $f:[0,1] \rightarrow \mathbb{R}$ is smooth and strictly monotone. A twist map is called positive/negative, if $f$ is increasing/decreasing.

(3) Let $N^{\prime}$ and $\ell^{\prime}$ be as in (2). If $\ell^{\prime}=1$ and $\phi \mid N^{\prime}$ is a twist map, then $\operatorname{im}(f) \subset[0,1]$, i.e. $\phi \mid \operatorname{int}\left(N^{\prime}\right)$ has no fixed points.

(4) If two connected components of $N$ are homotopic, then the corresponding local models of $\phi$ are either both positive or both negative twists.

Remarks. (i) Let $\phi$ be a diffeomorphism of finite type and $\ell$ be as in (1). Then $\phi^{\ell}$ is the product of (multiple) Dehn twists "along $N$ ". Moreover, two parallel Dehn twists have the same sign, by (4). We say 
that $\phi$ has uniform twists if $\phi^{\ell}$ is the product of only positive, or only negative Dehn twists.

(ii) A mapping class of $\Sigma$ is called algebraically finite if it does not have any pseudo-Anosov components in the sense of Thurston's theory of surface diffeomorphism. Every such class is represented by a diffeomorphism of finite type.

To see this, recall Thurston's classification theorem, [30, Theorem 4]: for every mapping class of $\Sigma$, there exists a diffeomorphism $\phi$ representing the class and a $\phi$-invariant finite union $C \subset \Sigma$ of non-contractible disjoint circles such that:

$\left(1^{\prime}\right)$ The components of $C$ are pairwise non-homotopic,

$\left(2^{\prime}\right)$ If $\Sigma^{\prime}$ is a $\phi$-invariant union of connected components of $\Sigma \backslash C$, then $\phi \mid \Sigma^{\prime}$ is isotopic to either a periodic or a pseudo-Anosov map.

The set $C$ is called a reducing set. Starting with a mapping class without pseudo-Anosov components, one first chooses a minimal reducing set $C$, meaning that it has the minimal number of components of all reducing sets. Minimality guarantees that after isotopying the NielsenThurston representative $\phi$ on a complement of a tubular neighborhood $N$ of $C$ to a periodic map, $\phi \mid N$ does not have periodic components. One can thus achieve condition (2) above, by isotopying $\phi \mid N$ relative to $\partial N$. If $(3)$ is not satisfied, this is achieved in a last step by introducing further components of $C$, violating $\left(1^{\prime}\right)$, but such that (4) still holds.

(iii) The term algebraically finite goes back to Nielsen [22]. Fried [12] defined the notion of algebraically finite diffeomorphism in any dimension. In two dimensions, these are special representatives of algebraically finite mapping classes. Fried's definition, however, is adopted to the theory of dynamical systems. For our purpose, a representative which is of the special type defined above is most convenient.

(iv) The term flip-twist map is taken from [16].

The rest of this section is devoted to the study of diffeomorphisms of finite type. The points of interest for Floer homology are: fixed point classes, monotonicity and action. The results we obtain are used in Section 5 to compute the Floer homology.

Convention. From now on, $\phi$ denotes a diffeomorphism of finite type and $N$ the associated $\phi$-invariant union of annuli. By $\Sigma_{0}$ we denote the union of the components of $\Sigma \backslash \operatorname{int}(N)$, where $\phi$ restricts to the identity. 
Furthermore, we denote by $\ell$ the smallest positive integer such that $\phi^{\ell}$ restricts to the identity on $\Sigma \backslash N$.

The first proposition describes the set of fixed point classes of $\phi$. It is a special case of a theorem by B. Jiang and J. Guo [16], which gives for any mapping class a representative that realizes its Nielsen number.

Recall that two fixed points $x$ and $y$ of $\phi$ are called equivalent if the following holds: there exists a continuous path $\gamma$ in $\Sigma$ connecting $x$ and $y$, such that $\gamma$ and $\phi \circ \gamma$ are homotopic relative to the endpoints. An equivalence class of fixed points is called a fixed point class.

Proposition 9 (Fixed point classes). Each fixed point class of $\phi$ is either a connected component of $\Sigma_{0}$ or consists of a single fixed point. $A$ fixed point $x$ of the second type satisfies $\operatorname{det}\left(\mathrm{id}-\mathrm{d} \phi_{x}\right)>0$.

The crucial step in our proof of this proposition is to prove it in the special case of products of disjoint Dehn twists. For this, we refer to Appendix A.

Proof of Proposition 9. First note that if $x \in \operatorname{Fix}(\phi) \cap \operatorname{int}(N)$, then $\phi$ restricted to the component of $N$ containing $x$, is a flip-twist map and $x=\left(\frac{1}{2},-\frac{1}{2} f\left(\frac{1}{2}\right)\right)$ or $\left(\frac{1}{2}, \frac{1}{2}-\frac{1}{2} f\left(\frac{1}{2}\right)\right)$. Now let $x \neq y$ be arbitrary fixed points in the same fixed point class. We prove in three steps that $x$ and $y$ are in the same connected component of $\Sigma_{0}$.

Claim 1. $x$ and $y$ are in the same connected component of either $\operatorname{int}(N)$ or $\Sigma \backslash \operatorname{int}(N)$.

Note that every connected component of $\Sigma \backslash \operatorname{int}(N)$ is a connected component of Fix $\left(\phi^{\ell}\right)$. Similarly, if $x \in \operatorname{int}(N)$, then $x$ is contained in a fixed circle of $\phi^{\ell}$. Such a circle is also a connected component of $\operatorname{Fix}\left(\phi^{\ell}\right)$. By Corollary 21 in Appendix A, however, every connected component of $\operatorname{Fix}\left(\phi^{\ell}\right)$ is a fixed point class of $\phi^{\ell}$. Since $x$ and $y$ are in the same fixed point class of $\phi^{\ell}$, this proves claim 1 . Denote by $M$ the connected component of $N$ or $\Sigma \backslash \operatorname{int}(N)$, containing $x$ and $y$.

Claim 2. $x$ and $y$ are in the same fixed point class of $\phi \mid M$.

By assumption, there exists a map $u:[0,1]^{2} \rightarrow \Sigma$ with

$$
u(0, t)=x, \quad u(1, t)=y \quad \text { and } \quad u(s, 1)=\phi(u(s, 0)),
$$

for all $s, t \in[0,1]$. By Corollary 21 , we can assume that $u(s, 0) \in M$, for all $s \in[0,1]$. Let $M^{\prime}$ be the union of $M$ and a tubular neighborhood of $\partial M$. We prove that $u$ can be deformed in the interior of $[0,1]^{2}$ such that its image is contained in $M^{\prime}$. First note that by a small perturbation, we may assume that $u$ is transverse to $\partial M^{\prime}$. Hence $u^{-1}\left(\partial M^{\prime}\right) \subset[0,1]^{2}$ is 
a 1-dimensional submanifold with boundary $\partial u^{-1}\left(\partial M^{\prime}\right)=u^{-1}\left(\partial M^{\prime}\right) \cap$ $\partial[0,1]^{2}=\emptyset$. Every component of $u^{-1}\left(\partial M^{\prime}\right)$ is therefore a circle and bounds a disk in $[0,1]^{2}$. The restriction of $u$ to such a disk represents an element of $\pi_{2}\left(\Sigma, \partial M^{\prime}\right)$. Since $\pi_{2}\left(\Sigma, \partial M^{\prime}\right)=0, u$ can be deformed in the interior of $[0,1]^{2}$ to a map $v$ such that the number of components of $v^{-1}\left(\partial M^{\prime}\right)$ is less than that of $u^{-1}\left(\partial M^{\prime}\right)$. It follows inductively that $u$ can be deformed in the interior of $[0,1]^{2}$ to a map $w$ with $w^{-1}\left(\partial M^{\prime}\right)=\emptyset$. This proves claim 2 and we are left with

Claim 3. Let $M$ be a compact connected oriented 2-manifold with boundary of Euler characteristic $\leq 0$. Let $\varphi$ be either a flip-twist map or a non-trivial orientation preserving periodic diffeomorphism of $M$. Then each fixed point class of $\varphi$ consists of a single point.

For a flip-twist map, this is checked explicitly by using the model. The other case was first proven in [15]. We repeat the argument here. First assume that $M$ is closed. The uniformization theorem states that in every conformal class of metrics on $M$, there is a unique metric of constant curvature -1 if $\chi(M)<0$ or 0 if $\chi(M)=0$. This implies that the unique representative of a $\varphi$-invariant conformal class, such a class exists since $\varphi$ is finite order, is itself $\varphi$-invariant. Hence we can pick a $\varphi$-invariant metric of constant curvature -1 or 0 on $M$ and lift $\varphi$ to an isometry $\tilde{\varphi}$ of the universal cover $\tilde{M}$ of $M . \tilde{M}$ is either isometric to the hyperbolic plane $\mathbb{H}^{2}$ or the Euclidean plane $\mathbb{R}^{2}$.

Let $x \in \operatorname{Fix}(\varphi)$ and let $\tilde{\varphi}, \tilde{x}$ be lifts of $\varphi, x$ to $\tilde{M}$, such that $\tilde{\varphi}(\tilde{x})=\tilde{x}$. Note that a fixed point of $\varphi$ is in the same class as $x$ if and only if it can be lifted to a fixed point of $\tilde{\varphi}$. Assume by contradiction that $\tilde{y} \neq \tilde{x}$ is a fixed point of $\tilde{\varphi}$. It follows that the unique geodesic going through $\tilde{x}$ and $\tilde{y}$ is pointwise fixed by $\tilde{\varphi}$. In particular, since $\tilde{\varphi}$ preserves orientation, $\mathrm{d} \tilde{\varphi}_{\tilde{x}}=\mathrm{id}$. This implies that $\tilde{\varphi}=\mathrm{id}$, because an isometry of $\mathbb{H}^{2}$ or $\mathbb{R}^{2}$ is determined by its value and differential at one point. This proves claim 3 in the case that $M$ is closed.

The case $\partial M \neq \emptyset$ is reduced to the above case by gluing two copies of $M$ together along a $\varphi$-invariant tubular neighborhood of $\partial M$. The glued manifold is closed and of Euler characteristic $\leq 0 ; \varphi$ extends to a non-trivial diffeomorphism $\varphi^{\prime}$, which is orientation preserving and of finite order. Hence, every fixed point class of $\varphi^{\prime}$ is a single point. The same therefore holds for $\varphi$. This ends the proof of claim 3. Finally we have

Claim 4. If $x \in \operatorname{Fix}(\phi) \backslash \Sigma_{0}$, then $\operatorname{det}\left(\mathrm{id}-\mathrm{d} \phi_{x}\right)>0$.

The point $x$ is a fixed point of either a flip-twist map or an orientation 
preserving non-trivial isometry. In the first case, the assertion is checked by using the local model. Similarly, one checks in the second case that $\operatorname{det}\left(\mathrm{id}-\mathrm{d} \phi_{x}\right) \leq 0$ if and only if $\mathrm{d} \phi_{x}=\mathrm{id}$. As shown in the proof of claim 3 , however, $\mathrm{d} \phi_{x}=\mathrm{id}$ implies that $x \in \Sigma_{0}$, which is a contradiction.

The next issue is monotonicity. First note that if $\omega^{\prime}$ is an area form on $\Sigma$ which is the standard form $\mathrm{d} q \wedge \mathrm{d} p$ with respect to the $(q, p)$ coordinates on $N$, then $\omega:=\sum_{i=1}^{\ell}\left(\phi^{i}\right)^{*} \omega^{\prime}$ is standard on $N$ and $\phi$ invariant, i.e. $\phi \in \operatorname{Symp}(\Sigma, \omega)$. To prove that $\omega$ can be chosen such that $\phi \in \operatorname{Symp}^{m}(\Sigma, \omega)$, we distinguish two cases: uniform and non-uniform twists. In the first case we have the following stronger statement.

Proposition 10 (Monotone 1). If $\phi$ has uniform twists and $\omega$ is a $\phi$-invariant area form, then $\phi \in \operatorname{Symp}^{m}(\Sigma, \omega)$.

Proof. By Lemma 7, it is enough to prove that $\phi^{\ell}$ is monotone with respect to any $\phi$-invariant area form. Replace $\phi$ by $\phi^{\ell}$. By the uniform twist condition, $\phi$ is the product of disjoint Dehn twists which are all, say positive. We prove that $\phi$ satisfies the hypothesis of Lemma 6 and is therefore monotone. We use the Picard-Lefschetz formula for the action of a positive Dehn twist on $H_{1}(\Sigma ; \mathbb{Z})$ : if the twist is along $C \subset \Sigma$, then $\alpha \mapsto \alpha-(\alpha \cdot[C])[C]$, where $\alpha \in H_{1}(\Sigma ; \mathbb{Z})$ and $[C]$ denotes the homology class of $C$ with respect to some orientation. The dot stands for the intersection pairing.

Let $C_{1}, \ldots, C_{n} \subset \Sigma$ be the disjoint non-contractible circles along which $\phi$ twists. Choose orientations of the $C_{i}$. Let $\alpha \in \operatorname{ker}\left(\mathrm{id}-\phi_{*}\right) \subset$ $H_{1}(\Sigma ; \mathbb{Z})$. We claim that for all $i=1, \ldots, n, \alpha \cdot\left[C_{i}\right]=0$. This is equivalent to the condition that $\alpha$ is represented by a map $S \rightarrow \operatorname{Fix}(\phi)$, where $S$ is a compact oriented 1-manifold, and therefore ends the proof of the proposition. Since the $C_{i}$ are pairwise disjoint, it follows from the Picard-Lefschetz formula that

$$
\alpha=\phi_{*} \alpha=\alpha-\sum_{i=1}^{n}\left(\alpha \cdot\left[C_{i}\right]\right)\left[C_{i}\right]
$$

and hence that $\sum_{i=1}^{n}\left(\alpha \cdot\left[C_{i}\right]\right)\left[C_{i}\right]=0$. Pairing with $\alpha$, we get $\sum_{i=1}^{n}(\alpha \cdot$ $\left.\left[C_{i}\right]\right)^{2}=0$, which implies that $\alpha \cdot\left[C_{i}\right]=0$, for all $i=1, \ldots, n$.

In the non-uniform case, monotonicity is a more subtle point and does not hold for arbitrary $\phi$-invariant area forms.

Proposition 11 (Monotone 2). If $\phi$ does not have uniform twists, there exists a $\phi$-invariant area form $\omega$ such that $\phi \in \operatorname{Symp}^{m}(\Sigma, \omega)$. 
Moreover, $\omega$ can be chosen such that it is the standard form $\mathrm{d} q \wedge \mathrm{d} p$ on $N$.

Proof. The strategy of the proof is the following. Assume first that $\phi \mid \Sigma \backslash N=$ id. We begin by defining a $\phi$-invariant area form $\omega$ with $\operatorname{area}_{\omega}(\Sigma)=-\chi(\Sigma)$. Then we construct for every class $[\gamma] \in \operatorname{ker}\left(\mathrm{id}-\phi_{*}\right)$, a class $[\Gamma] \in H_{2}\left(T_{\phi} ; \mathbb{Z}\right)$ such that

$$
\hat{\partial}[\Gamma]=[\gamma] \quad \text { and } \quad\left\langle\left[\omega_{\phi}\right],[\Gamma]\right\rangle=-\left\langle c_{\phi},[\Gamma]\right\rangle
$$

with $\hat{\partial}$ as defined in the sequence (5). Finally, we show how the general case is reduced to the case above.

We start with the following set-up. Let $\tilde{N} \subset \Sigma$ be a union of disjoint non-contractible and pairwise non-homotopic annuli such that $\phi \mid \Sigma \backslash$ $\tilde{N}=$ id. Let for every connected component $N^{\prime}$ of $\tilde{N}, \ell^{\prime}$ be a positive integer and $f:[0,1] \rightarrow \mathbb{R}$ be a smooth monotone function with $f(0)=$ $0, f(1)=\ell^{\prime}$ and such that $\phi \mid N^{\prime}$ is (an $\ell^{\prime}$-fold Dehn twist) given by the model $(q, p) \mapsto(q, p-f(q))$ for $(q, p) \in[0,1] \times S^{1}$. Note that if two connected components of $N$ are homotopic, they are contained in the same connected component of $\tilde{N}$. We emphasize here that not only the function $f$ but also the local chart of $N^{\prime}$ is fixed for the rest of the proof.

In a first step, we choose for every component $N^{\prime}$ of $\tilde{N}$, an embedded circle $C^{\prime} \subset N^{\prime}$ as follows. Look at the set $\operatorname{graph}(-f) \subset[0,1] \times\left[-\ell^{\prime}, 0\right]$ if $\ell^{\prime}>0$, respectively $[0,1] \times\left[0,-\ell^{\prime}\right]$ if $\ell^{\prime}<0$. See the figures below.

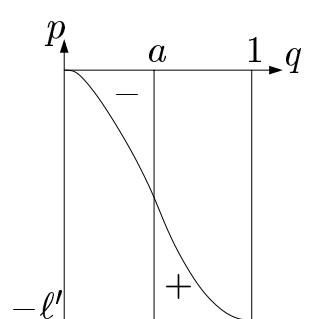

Figure 1: graph $(-f)$ for a positive twist

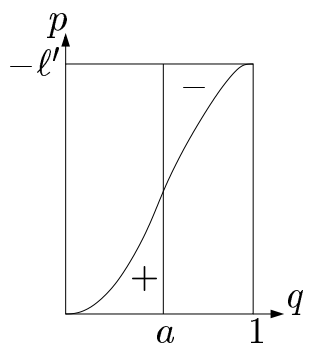

Figure 2: graph $(-f)$ for a negative twist

For any $a \in(0,1)$, the complement of the union of $\operatorname{graph}(-f)$ and the set $\{q=a\}$ has four components. If $\phi \mid N^{\prime}$ is a positive twist, we choose $a$ such that the left upper component (indicated with a - sign in Figure 1) and the right lower component (indicated with a + sign) have the same area with respect to the standard area form on $[0,1] \times\left[-\ell^{\prime}, 0\right]$. If $\phi \mid N^{\prime}$ is a negative twist, left upper is replaced by left lower and right 
lower by right upper and the signs are interchanged. In both cases, we set $C^{\prime}:=\{a\} \times S^{1} \subset N^{\prime}$, with orientation induced from $S^{1}$. The purpose of this construction will become clear below. Let $C$ denote the union of the loops $C^{\prime}$. Let $\Sigma_{1}, \ldots, \Sigma_{m} \subset \Sigma$ denote the closures of the connected components of $\Sigma \backslash C$. Since the $C^{\prime}$ are disjoint non-contractible and pairwise non-homotopic, it follows that $\chi\left(\Sigma_{j}\right)<0$ for all $j=1, \ldots, m$. Now choose an area form $\omega$ on $\Sigma$ such that

$$
\begin{aligned}
\operatorname{area}_{\omega}\left(\Sigma_{j}\right) & =-\chi\left(\Sigma_{j}\right) \quad \text { for all } j=1, \ldots, m, \\
\omega \mid \tilde{N} & =\varepsilon \cdot \mathrm{d} q \wedge \mathrm{d} p,
\end{aligned}
$$

where $\varepsilon>0$ is sufficiently small. By the first condition, we have that $\operatorname{area}_{\omega}(\Sigma)=-\chi(\Sigma)$ and from the second it follows that $\phi^{*} \omega=\omega$. We now prove in several steps that $\left[\omega_{\phi}\right]=-c_{\phi}$ in $H^{2}\left(T_{\phi} ; \mathbb{R}\right)$.

Let $S$ be a compact oriented 1-manifold and $\gamma: S \rightarrow \Sigma$ be an immersion which is transverse to $C$. Moreover, assume that $[\gamma]=[\phi \circ \gamma]$ in $H_{1}(\Sigma ; \mathbb{Z})$. The goal is to lift the 1 -cycle $\gamma$ to a 2 -cycle $\Gamma$ in $T_{\phi}$. For this, we first define a 2-chain $A$ in $\Sigma$ which satisfies

$$
\partial A=\gamma-\phi \circ \gamma-\sum_{i=1}^{n} \ell_{i}\left([\gamma] \cdot\left[C_{i}\right]\right) C_{i},
$$

where we think of the right hand side as a 1-chain. Here, we have introduced a numbering of the components of $C$. The chain $A$ can be described a follows, compare Figure 1, 2: At every intersection point of $\gamma$ and $C$ where $\gamma$ runs in the positive $q$-direction, there is a local contribution to $A$ given by the regions in Figure 1, 2 which are labelled by \pm . The sign of the contribution is as indicated in the figure. If $\gamma$ runs in the negative $q$-direction, the signs are interchanged. Note that by our choice of $\omega$, we have that $\int_{A} \omega=0$.

Next, we use that $\gamma$ is homologous to $\phi \circ \gamma$, i.e. that

$$
\sum_{i=1}^{n} \ell_{i}\left([\gamma] \cdot\left[C_{i}\right]\right)\left[C_{i}\right]=0 .
$$

This means that there exist integers $k_{1}, \ldots, k_{m}$ such that

$$
\partial\left(\sum_{j=1}^{m} k_{j} \Sigma_{j}\right)=\sum_{i=1}^{n} \ell_{i}\left([\gamma] \cdot\left[C_{i}\right]\right) C_{i} .
$$

We can now define the 2-chain $\Gamma$ in $T_{\phi}=[0,1] \times \Sigma /(0, \phi(x)) \sim(1, x)$, by

$$
\Gamma:=-[0,1 / 2] \times(\phi \circ \gamma)-\{1 / 2\} \times\left(A+\sum_{j=1}^{m} k_{j} \Sigma_{j}\right)-[1 / 2,1] \times \gamma .
$$


By construction, $\Gamma$ is a cycle; indeed

$$
\begin{aligned}
\partial \Gamma= & \{0\} \times(\phi \circ \gamma)-\{1 / 2\} \times(\phi \circ \gamma)-\{1 / 2\} \times \partial A \\
& -\{1 / 2\} \times \partial\left(\sum_{j=1}^{m} k_{j} \Sigma_{j}\right)+\{1 / 2\} \times \gamma-\{1\} \times \gamma \\
= & 0 .
\end{aligned}
$$

By a similar calculation as in the proof of Lemma 6 , it furthermore follows that $\hat{\partial}[\Gamma]=[\gamma]$.

Claim 1. $\left\langle\left[\omega_{\phi}\right],[\Gamma]\right\rangle=-\sum_{j=1}^{m} k_{j} \operatorname{area}_{\omega}\left(\Sigma_{j}\right)$.

Only the middle summand of $\Gamma$ contributes to $\left\langle\left[\omega_{\phi}\right],[\Gamma]\right\rangle$. Since $A$ has vanishing $\omega$-area, this already proves claim 1.

Claim 2. $\left\langle c_{\phi},[\Gamma]\right\rangle=-\sum_{j=1}^{m} k_{j} \chi\left(\Sigma_{j}\right)$.

To prove this, we use the following property of the Euler class. If a smooth section $s: T_{\phi} \rightarrow V_{\phi}$ is transverse to the zero-section, then $s^{-1}(0) \subset T_{\phi}$ is a submanifold of codimension 2 and its homology class, with respect to a suitable orientation, is Poincaré-dual to $c_{\phi}$. In particular, $\left\langle c_{\phi},[u]\right\rangle$ equals the intersection number $\left[s^{-1}(0)\right] \cdot[u]$, for any $[u] \in H_{2}\left(T_{\phi} ; \mathbb{Z}\right)$. The orientation of $s^{-1}(0)$ at a point $x$ is defined as follows. Let $\left\{e_{1}, e_{2}, e_{3}\right\}$ be an oriented basis of $T_{x} T_{\phi}$ such that $e_{1}$ is tangent to $s^{-1}(0)$. Then $e_{1}$ is said to be oriented if $\left\{e_{1}, e_{2}, e_{3}, \mathrm{~d} s_{x} e_{2}, \mathrm{~d} s_{x} e_{3}\right\}$ is an oriented basis of

$$
T_{(x, 0)} V_{\phi} \cong \mathbb{R} \oplus T_{x} \Sigma \oplus T_{x} \Sigma \text {. }
$$

We now define a smooth section of $V_{\phi}$. To begin with, we choose a vector field $\xi$ on $\Sigma$ with only non-degenerate zeros and such that $\xi \mid \tilde{N}=\partial / \partial q$. Furthermore, we require that $\xi^{-1}(0)$ is disjoint from $\operatorname{im}(\gamma)$. That such a vector field exists is a standard result in differential topology. By the Poincaré-Hopf Theorem, the sum of indices of $\xi$ over all zeros in $\Sigma_{j}$ equals $\chi\left(\Sigma_{j}\right)$, for all $j=1, \ldots, m$.

Note that the vector field $\phi^{*} \xi-\xi$ is supported in $\tilde{N}$, where it is given by $\left(0,-f^{\prime}(q)\right)$ with respect to the local model. Hence, there exists a smooth path $\left(\xi_{t}\right)_{t \in \mathbb{R}}$ of vector fields such that $\xi_{t+1}=\phi^{*} \xi_{t}, \xi_{t}=\xi$ on $\Sigma \backslash \tilde{N}$ and $\xi_{t}^{-1}(0)=\xi^{-1}(0)$. Let the section $s: T_{\phi} \rightarrow V_{\phi}$ be defined by $s([t, x]):=\left[t, \xi_{t}(x)\right]$; recall that

$$
V_{\phi}=\mathbb{R} \times T \Sigma /\left(t+1, \xi_{x}\right) \sim\left(t, \mathrm{~d} \phi_{x} \xi_{x}\right) .
$$


By our choice of the vector field $\xi, s$ in transverse to the zero-section and thus, $\left[s^{-1}(0)\right]$ is Poincaré-dual to $c_{\phi}$. Moreover,

$$
\begin{aligned}
{\left[s^{-1}(0)\right] \cdot[\Gamma] } & =-\sum_{j=1}^{m} k_{j}\left(s^{-1}(0) \cdot \Sigma_{j}\right) \\
& =-\sum_{j=1}^{m} k_{j} \cdot \chi\left(\Sigma_{j}\right) .
\end{aligned}
$$

The numbers $s^{-1}(0) \cdot \Sigma_{j}$ are well defined because $s^{-1}(0)$ intersects $\Sigma_{j}$ transversally and in the interior of $\Sigma_{j}$. Note that the sign of an intersection point equals the index of $\xi$ at that point. This proves claim 2 . From claim 1, 2 and the first equation in (7), we conclude that

$$
\left\langle\left[\omega_{\phi}\right],[\Gamma]\right\rangle=-\left\langle c_{\phi},[\Gamma]\right\rangle,
$$

and hence that $\phi \in \operatorname{Symp}^{m}(\Sigma, \omega)$. We end the proof of the proposition with the following observation. Let $\phi$ be a diffeomorphism of finite type; apply the above construction to $\phi^{\ell}$ and let $\omega$ be an area form which satisfies (7). It follows that

$$
\omega^{\prime}:=\frac{1}{\ell} \sum_{i=1}^{\ell}\left(\phi^{i}\right)^{*} \omega
$$

also satisfies (7) and hence that $\phi^{\ell} \in \operatorname{Symp}^{m}\left(\Sigma, \omega^{\prime}\right)$. On the other hand $\phi^{*} \omega^{\prime}=\omega^{\prime}$, and therefore $\phi \in \operatorname{Symp}^{m}\left(\Sigma, \omega^{\prime}\right)$, by Lemma 7 .

The idea of the proof is the following. As above, we first replace $\phi$ by $\phi^{\ell}$. Then we construct an $\phi$-invariant area form $\omega$ with $\operatorname{area}_{\omega}(\Sigma)=$ $-\chi(\Sigma)$. The main step is the following: for every integer class $[\gamma] \in$ $\operatorname{ker}\left(\mathrm{id}-\phi_{*}\right)$ we construct a class $[\Gamma] \in H_{2}\left(T_{\phi} ; \mathbb{Z}\right)$ such that

$$
\partial[\Gamma]=[\gamma] \quad \text { and } \quad\left\langle\left[\omega_{\phi}\right],[\Gamma]\right\rangle=-\left\langle c_{\phi},[\Gamma]\right\rangle \text {. }
$$

This proves the proposition for $\phi$. Finally, we show that $\omega$ can be chosen such that it is invariant under the original $\phi$. By Lemma 7 this proves the proposition.

Next we consider the symplectic action $\alpha_{\omega}$ on the twisted loop space $\Omega_{\phi}$ and prove that it is exact. See (3) for the definition of $\alpha_{\omega}$. This result is crucial for the computation of the Floer homology, in particular for the use of the connecting orbits proposition proved in the next section.

We need the following lemma, which holds for any $\phi \in \operatorname{Symp}(\Sigma, \omega)$. First note that a loop in $\Omega_{\phi}$ is represented by a map $u: S^{1} \times[0,1] \rightarrow \Sigma$ 
with

$$
u(s, t)=\phi(u(s, t+1)) \quad \text { for all }(s, t) \in S^{1} \times[0,1] .
$$

By $[u]$ we denote the homology class of the loop $u$ in $\Omega_{\phi}$.

Lemma 12. Let $u$ and $v$ be two loops in $\Omega_{\phi}$. If $u(., 0)$ and $v(., 0)$ are freely homotopic loops in $\Sigma$, then $\left\langle\left[\alpha_{\omega}\right],[u]\right\rangle=\left\langle\left[\alpha_{\omega}\right],[v]\right\rangle$.

Proof. Let $w: S^{1} \times[0,1] \rightarrow \Sigma$ be such that $w(., 0)=u(., 0)$ and $w(., 1)=v(., 0)$. Define the map $u^{\prime}:=w^{-1} \# u \#\left(\phi^{-1} \circ w\right): S^{1} \times[0,1] \rightarrow$ $\Sigma$ by

$$
u^{\prime}(s, t)= \begin{cases}w(s, 1-3 t) & \text { if } t \in[0,1 / 3] \\ u(s, 3 t-1) & \text { if } t \in[1 / 3,2 / 3] \\ \phi^{-1}(w(s, 3 t-2)) & \text { if } t \in[2 / 3,1] .\end{cases}
$$

Since for all $t \in[0,1 / 3], s \in S^{1}, u^{\prime}(s, 1-t)=\phi^{-1}(w(s, 1-3 t))=$ $\phi^{-1}\left(u^{\prime}(s, t)\right)$, it follows that $u^{\prime}$ and $u$ are homotopic loops in $\Omega_{\phi}$; in particular $[u]=\left[u^{\prime}\right]$. Note that since $u^{\prime}(s, 0)=v(s, 0)$ and $u^{\prime}(s, 1)=$ $v(s, 1)$ for all $s \in S^{1}$, the map $u^{\prime} \# v^{-1}$ descends to a map $S^{1} \times S^{1} \rightarrow \Sigma$. Now if $w$ is an arbitrary loop in $\Omega_{\phi}$, then

$$
\left\langle\left[\alpha_{\omega}\right],[w]\right\rangle=-\int_{S^{1} \times[0,1]} w^{*} \omega .
$$

Therefore

$$
\left\langle\left[\alpha_{\omega}\right],\left[u^{\prime}\right]\right\rangle-\left\langle\left[\alpha_{\omega}\right],[v]\right\rangle=-\int_{S^{1} \times S^{1}}\left(u^{\prime} \# v^{-1}\right)^{*} \omega=0 .
$$

In the last equality we use the fact that the mapping degree of $u^{\prime} \# v^{-1}$ vanishes, since the genus of $\Sigma$ is $\geq 2$. This proves the proposition.

We return to the situation where $\phi$ is a diffeomorphism of finite type. The proof of the following proposition relies on the results discussed in Appendix A.

Proposition 13 (Action). If $\omega$ is a $\phi$-invariant area form, then $\alpha_{\omega}$ has vanishing periods.

Proof. For any loop $u$ in $\Omega_{\phi}$, define $v: S^{1} \times[0, \ell] \rightarrow \Sigma$ by

$$
v(s, t)=\phi^{-j}(u(s, t-j)) \quad \text { for } \quad(s, t) \in S^{1} \times[j, j+1], j<\ell .
$$

Since $v(s, 0)=\phi^{\ell}(v(s, \ell))$ for all $s \in S^{1}, v$ can be considered a loop in $\Omega_{\phi^{\ell}}$. Note that

$$
-\int_{S^{1} \times[0, \ell]} v^{*} \omega=\ell \cdot\left\langle\left[\alpha_{\omega}\right],[u]\right\rangle .
$$


Now observe that $v(., 0)$ is freely homotopic to $\phi^{-1}(v(., 0))=v(., 1)$. By Corollary 21 in Appendix A, we therefore know that $v(., 0)$ is freely homotopic to a loop $\gamma: S^{1} \rightarrow \operatorname{Fix}\left(\phi^{\ell}\right)$. Hence, it follows from the previous lemma with $\phi$ replaced by $\phi^{\ell}$ that

$$
\int_{S^{1} \times[0, \ell]} v^{*} \omega=\int_{S^{1}} \gamma^{*} \omega=0 .
$$

Therefore $\left\langle\left[\alpha_{\omega}\right],[u]\right\rangle=0$, which ends the proof of the proposition.

\section{Connecting orbits}

The main result of this section is a separation mechanism for Floer connecting orbits. Together with the topological separation of fixed points discussed in Proposition 9, it allows us to compute the Floer homology of diffeomorphisms of finite type. We expect, however that the results of this section are applicable to a larger class of surface diffeomorphisms.

We start by introducing the setup. The notation is reminiscent of the situation encountered in Section 3. However, it is only in the next section that we return our attention to diffeomorphisms of finite type.

Let $\Sigma_{0} \subset \Sigma$ be a compact submanifold, not necessarily connected. Let $N_{0} \subset \Sigma_{0}$ be a collar neighborhood of $\partial \Sigma_{0}$. On every connected component of $N_{0}$, we choose coordinates $(q, p) \in[0,1] \times S^{1}$ such that $\partial \Sigma_{0} \cong \bigcup\{1\} \times S^{1}$. Let $\omega$ be an area form on $\Sigma$ which is given by $\mathrm{d} q \wedge \mathrm{d} p$ on $N_{0}$. Let $\Phi \in \operatorname{Symp}(\Sigma, \omega), H: \Sigma \rightarrow \mathbb{R}$ a smooth function and $J=\left(J_{t}\right)_{t \in \mathbb{R}}$ such that the following holds: (H1) $\Sigma_{0}$ is $\Phi$-invariant. Moreover, $\Phi(x)=\psi_{1}(x)$ for all $x \in \Sigma_{0}$, where $\left(\psi_{t}\right)_{t \in \mathbb{R}}$ denotes the Hamiltonian flow generated by $H$. This means that $\partial_{t} \psi_{t}=X \circ \psi_{t}$, where the vector field $X$ is defined by $\mathrm{d} H=\omega(X, \cdot)$.

(H2) There exists a constant $0<\delta<1 / 4$ such that on each connected component of $N_{0}$, we have $\Phi(q, p)=(q, p \mp \delta)$. The sign may depend on the component.

(H3) $\operatorname{Fix}(\Phi) \cap \Sigma_{0}=\operatorname{Crit}(H) \cap \Sigma_{0}$.

(H4) If $\omega^{\prime}$ is a $\Phi$-invariant area form such that $\omega^{\prime}=\omega$ on $\Sigma \backslash N_{0}$, then $\alpha_{\omega^{\prime}}$ has vanishing periods on $\Omega_{\Phi}$.

(H5) For all $t \in \mathbb{R}, J_{t}$ is an $\omega$-compatible complex structure which restricts to the standard complex structure on $N_{0}$ with respect to the $(q, p)$-coordinates. Moreover, $J_{t+1}=\Phi^{*} J_{t}$.

Assuming (H1-5) we prove that 
Proposition 14 (Connecting orbits). Let $x^{-}, x^{+} \in \operatorname{Fix}(\Phi) \cap \Sigma_{0}$ be in the same connected component of $\Sigma_{0}$. If $u \in \mathcal{M}\left(x^{-}, x^{+} ; J\right.$, $\left.\Phi\right)$, then $\operatorname{im} u \subset \Sigma_{\delta}$, where $\Sigma_{\delta}$ denotes the $\delta$-neighborhood of $\Sigma_{0} \backslash N_{0}$ with respect to any of the metrics $\omega\left(., J_{t}.\right)$.

To prove this proposition we vary the symplectic form. Fix $\varepsilon>0$ sufficiently small and set $N_{\varepsilon}:=\bigcup[\varepsilon, 1-\varepsilon] \times S^{1} \subset N_{0}$. For every $R>0$, let $\lambda_{R}: \Sigma \rightarrow \mathbb{R}_{>0}$ be a $\Phi$-invariant smooth function such that

$$
\lambda_{R} \equiv \begin{cases}R & \text { on } N_{\varepsilon} \\ 1 & \text { on } \Sigma \backslash N_{0}\end{cases}
$$

Set

$$
\omega_{R}:=\lambda_{R}^{2} \cdot \omega \text { and } \quad g_{R, t}:=\omega_{R}\left(\cdot, J_{t}\right) .
$$

Note that $\omega_{R}$ is $\Phi$-invariant and $\omega_{R}=\omega$ on $\Sigma \backslash N_{0}$. By (H4), we can define an action functional $\mathcal{A}_{R}: \Omega_{\Phi} \rightarrow \mathbb{R}$ such that

$$
\begin{aligned}
\mathcal{A}_{R}\left(y^{\prime}\right)-\mathcal{A}_{R}(y) & =\int_{0}^{1} \alpha_{\omega_{R}}(u(s, \cdot)) \partial_{s} u(s, \cdot) \mathrm{d} s \\
& =\int_{0}^{1} \int_{0}^{1} \omega_{R}\left(\partial_{t} u(s, t), \partial_{s} u(s, t)\right) \mathrm{d} t \mathrm{~d} s
\end{aligned}
$$

for all $y, y^{\prime} \in \Omega_{\Phi}$ and $u:[0,1] \times \mathbb{R} \rightarrow \Sigma$ with $u(s, t)=\Phi(u(s, 1+t))$ and $u(0, \cdot)=y, u(1, \cdot)=y^{\prime}$. The following observation is crucial for the proof of the proposition.

Lemma 15. Let $x^{-}, x^{+} \in \operatorname{Fix}(\Phi) \cap \Sigma_{0}$ be in the same connected component of $\Sigma_{0}$. Then

$$
\mathcal{A}_{R}\left(x^{+}\right)-\mathcal{A}_{R}\left(x^{-}\right)=H\left(x^{-}\right)-H\left(x^{+}\right),
$$

for every $R>0$.

Proof. Choose a path $\gamma:[0,1] \rightarrow \Sigma_{0} \backslash N_{0}$ from $x^{-}$to $x^{+}$and define $h:[0,1] \times \mathbb{R} \rightarrow \Sigma$ by

$$
h(s, t):=\psi_{-t}(\gamma(s))
$$


By (H1), we have $h(s, t)=\Phi(h(s, t+1))$ and furthermore, by (H3), $h(0,)=.x^{-}, h(1,)=.x^{+}$. Hence,

$$
\begin{aligned}
\mathcal{A}_{R}\left(x^{+}\right)-\mathcal{A}_{R}\left(x^{-}\right) & =\int_{0}^{1} \int_{0}^{1} \omega_{R}\left(\partial_{t} h(s, t), \partial_{s} h(s, t)\right) \mathrm{d} s \mathrm{~d} t \\
& =-\int_{0}^{1}\left(\int_{0}^{1} \mathrm{~d} H(h(s, t)) \partial_{s} h(s, t) \mathrm{d} s\right) \mathrm{d} t \\
& =\int_{0}^{1}(H(h(0, t))-H(h(1, t))) \mathrm{d} t \\
& =H\left(x^{-}\right)-H\left(x^{+}\right) .
\end{aligned}
$$

In the second line we use that

$$
\omega_{R}\left(., \partial_{t} h(s, t)\right)=\omega\left(., \partial_{t} h(s, t)\right)=\mathrm{d} H(h(s, t)),
$$

since $\operatorname{im}(h) \subset \Sigma_{0} \backslash N_{0}$.

Proof of Proposition 14. Let $u \in \mathcal{M}\left(x^{-}, x^{+} ; J, \Phi\right)$, i.e. $u: \mathbb{R}^{2} \rightarrow \Sigma$ is a smooth function satisfying

$$
\left\{\begin{array}{l}
u(s, t)=\phi(u(s, t+1)) \\
\partial_{s} u+J_{t}(u) \partial_{t} u=0 \\
\lim _{s \rightarrow \pm \infty} u(s, t)=x^{ \pm}
\end{array}\right.
$$

and

$$
\int_{\mathbb{R}} \int_{0}^{1} \omega\left(\partial_{t} u(s, t), J_{t} \partial_{t} u(s, t)\right) \mathrm{d} t \mathrm{~d} s<\infty .
$$

We prove that

$$
\operatorname{im}(u) \cap N_{\varepsilon}^{\prime}=\emptyset,
$$

where $N_{\varepsilon}^{\prime}=\bigcup(\varepsilon+\delta, 1 / 2] \times S^{1} \subset N_{0}$. Assume by contradiction that there exist $s_{1}<s_{2}$ and $t^{\prime}$ such that $u\left(s, t^{\prime}\right) \in N_{\varepsilon}^{\prime}$ for all $s \in\left[s_{1}, s_{2}\right]$.

Denote by $g$ the standard Euclidean metric on $N_{0}$. Note that if $x \in N_{\varepsilon}^{\prime}$, then $B_{g}(x, \delta) \subset N_{\varepsilon}$. Here $B_{g}(x, \delta)$ denotes the $g$-disk of radius $\delta$ around $x$. Moreover if $x=(q, p)$, then $\Phi^{-1}(x)=(q, p \pm \delta) \in \partial B_{g}(x, \delta)$.

Hence, it follows from the first equation in (8) that $u\left(s, t^{\prime}+1\right) \in$ $\partial B_{g}\left(u\left(s, t^{\prime}\right)\right)$ for all $s \in\left[s_{1}, s_{2}\right]$. Fix $s \in\left[s_{1}, s_{2}\right]$ and let $r \in(0,1]$ be such that

$$
u\left(\{s\} \times\left[t^{\prime}, t^{\prime}+r\right]\right) \subset B_{g}\left(u\left(s, t^{\prime}\right), \delta\right), \quad u\left(s, t^{\prime}+r\right) \in \partial B_{g}\left(u\left(s, t^{\prime}\right), \delta\right) .
$$

This implies that

$$
\delta \leq \int_{t^{\prime}}^{t^{\prime}+r}\left|\partial_{t} u(s, t)\right|_{g} \mathrm{~d} t
$$


By our choice of $J_{t}$, we know that $g_{R, t}=R^{2} g$ on $N_{\varepsilon}$, and hence that $R \cdot\left|\partial_{t} u(s, t)\right|_{g}=\left|\partial_{t} u(s, t)\right|_{g_{R, t}}$ for all $t \in\left[t^{\prime}, t^{\prime}+r\right]$. Therefore,

$$
\begin{aligned}
R \cdot \delta & \leq \int_{t^{\prime}}^{t^{\prime}+r}\left|\partial_{t} u(s, t)\right|_{g_{R, t}} \mathrm{~d} t \\
& \leq \int_{t^{\prime}}^{t^{\prime}+1}\left|\partial_{t} u(s, t)\right|_{g_{R, t}} \mathrm{~d} t=\int_{0}^{1}\left|\partial_{t} u(s, t)\right|_{g_{R, t}} \mathrm{~d} t .
\end{aligned}
$$

In the last step we use that $\left|\partial_{t} u(s, t)\right|_{g_{R, t}}$ is a 1-periodic function in $t$. By Hölder's inequality we get that

$$
R^{2} \cdot \delta^{2} \leq \int_{0}^{1}\left|\partial_{t} u(s, t)\right|_{g_{R, t}}^{2} \mathrm{~d} t
$$

for all $R>0$ and $s \in\left[s_{1}, s_{2}\right]$. Now integrate over $\left[s_{1}, s_{2}\right]$ :

$$
\begin{aligned}
R^{2} \cdot \delta^{2} \cdot\left|s_{1}-s_{2}\right| & \leq \int_{s_{1}}^{s_{2}} \int_{0}^{1}\left|\partial_{t} u(s, t)\right|_{g_{R, t}}^{2} \mathrm{~d} s \mathrm{~d} t \\
& \leq \int_{\mathbb{R}} \int_{0}^{1}\left|\partial_{t} u(s, t)\right|_{g_{R, t}}^{2} \mathrm{~d} s \mathrm{~d} t .
\end{aligned}
$$

Finally, we use the energy identity

$$
\left|\partial_{t} u(s, t)\right|_{g_{R, t}}^{2}=\omega_{R}\left(\partial_{s} u(s, t), \partial_{t} u(s, t)\right)
$$

which follows from the second equation in (8). Note that since $u$ has finite energy with respect to $\omega_{R}$, the energy identity implies that

$$
\int_{\mathbb{R}} \int_{0}^{1}\left|\partial_{t} u(s, t)\right|_{g_{R, t}}^{2} \mathrm{~d} s \mathrm{~d} t=\mathcal{A}_{R}\left(x^{-}\right)-\mathcal{A}_{R}\left(x^{+}\right) .
$$

From (9) and Lemma 15 we therefore get

$$
R^{2} \cdot \delta^{2} \cdot\left|s_{1}-s_{2}\right| \leq H\left(x^{+}\right)-H\left(x^{-}\right),
$$

for all $R>0$. For large $R$ this is a contradiction and proves that $\operatorname{im}(u)$ is disjoint from $N_{\varepsilon}^{\prime}$. Since $\varepsilon$ can be chosen arbitrarily small by an appropriate choice of functions $\lambda_{R}, \operatorname{im}(u)$ is disjoint from $\bigcup(\delta, 1 / 2] \times S^{1}$. This proves the proposition.

Remark. The advantage of our approach to the connecting orbits proposition compared to Seidel's original approach in [26] is that we do not have to make the function $H$ depend on $R$. Moreover, the bubbling argument completely disappears. This is relevant in the case where $\Phi$ twists with different signs at different "ends" of $\Sigma_{0}$. In that case, when the ends are stretched as in [26, Lemma 4], the energy difference 
of certain fixed points may go to infinity and the bubbling argument fails.

\section{Floer homology of finite type diffeomorphisms}

In this section we prove Theorem 1. We return to the notation of Section 3, i.e. $\phi$ is a diffeomorphism of finite type and $\Sigma_{0}$ denotes the union of connected components of $\Sigma \backslash \operatorname{int}(N)$, where $\phi$ restricts to the identity.

(Monotonicity) By Propositions 10 and 11 we can choose an area form $\omega$ such that $\phi \in \operatorname{Symp}^{m}(\Sigma, \omega)$. Note that every Hamiltonian perturbation of $\phi$ is also in $\operatorname{Symp}^{m}(\Sigma, \omega)$. We impose an additional condition on $\omega$ in the next paragraph.

(Hamiltonian perturbation) As a preparation, let $f_{1}, f_{2}:[0,3] \rightarrow \mathbb{R}$ be two functions which are constant on $[0,1]$ and such that $f_{1}(q)=f_{2}(q)$ for all $q \in[2,3]$. Define the function

$$
h(q)=\int_{q}^{3}\left(f_{1}(r)-f_{2}(r)\right) \mathrm{d} r,
$$

for $q \in[0,3]$. It follows that

$$
h(q)= \begin{cases}\delta \cdot q+c & \text { if } q \in[0,1] \\ 0 & \text { if } q \in[2,3]\end{cases}
$$

where $\delta=f_{2}(0)-f_{1}(0)$ and $c=\int_{0}^{3}\left(f_{1}(r)-f_{2}(r)\right) \mathrm{d} r$. Now consider $h(q)$ as a function on $[0,3] \times S^{1}$ with coordinates $(q, p)$. The Hamiltonian vector field of $h$ with respect to $\mathrm{d} q \wedge \mathrm{d} p$ is simply $\left(f_{1}(q)-f_{2}(q)\right) \cdot \partial / \partial p$ at the point $(q, p)$. The time-1-map of the flow is thus the twist map $(q, p) \mapsto\left(q, p+f_{1}(q)-f_{2}(q)\right)$. In particular, $(q, p) \mapsto(q, p-\delta)$ if $q \in[0,1]$.

This has the following application. Let $\tilde{N} \subset \Sigma$ be a $\phi$-invariant closed tubular neighborhood of $\partial \Sigma_{0}$. On every connected component of $\tilde{N}$, we choose coordinates $(q, p) \in[0,3] \times S^{1}$ such that $\phi$ is given by $(q, p) \mapsto$ $(q, p \mp f(q))$, for some monotone increasing $f:[0,3] \rightarrow[0,1)$. Moreover, we assume that $N_{0}:=\Sigma_{0} \cap \tilde{N} \cong \bigcup[0,1] \times S^{1}$. Note that $f \mid[0,1] \equiv 0$. Furthermore note that we can assume that $\omega=\mathrm{d} q \wedge \mathrm{d} p$ on $\tilde{N}$. It now follows from the preliminary remarks that for every $0<\delta<1 / 4$ there exists $h: \tilde{N} \rightarrow \mathbb{R}$ such that

(i) On a connected component of $N_{0}, h(q, p)= \pm \delta \cdot q+c$. Here, the sign and the constant $c$ may depend on the component. 
(ii) $h \equiv 0$ on $\bigcup[2,3] \times S^{1}$.

(iii) Let $\psi$ denote the time-1-map of the Hamiltonian flow generated by $h$ with respect to $\mathrm{d} q \wedge \mathrm{d} p$. For every connected component of $N_{0}$, there exists a monotone increasing function $g:[0,3] \rightarrow[\delta, 1)$, such that $\phi \circ \psi(q, p)=(q, p \mp g(q))$.

As a consequence of (i) and (ii), there exists a function $H: \Sigma \rightarrow \mathbb{R}$ with

$$
H(x)= \begin{cases}h(x) & \text { if } x \in \tilde{N} \\ 0 & \text { if } x \in \Sigma \backslash\left(\Sigma_{0} \cup \tilde{N}\right),\end{cases}
$$

and such that $H \mid \operatorname{int}\left(\Sigma_{0}\right)$ is a Morse function, meaning that all the critical points are non-degenerate. We refer to [24, Lemma 4.15] for the extension of Morse functions.

Let $\left(\psi_{t}\right)_{t \in \mathbb{R}}$ denote the Hamiltonian flow generated by $H$ with respect to the fixed area form $\omega$ and set

$$
\Phi:=\phi \circ \psi_{1} .
$$

By construction, $\omega, \Phi, H$ and $N_{0}$ satisfy the hypothesis $(H 1,2)$ of the last section. Moreover, by choosing $H$ and $\delta$ such that the $C^{2}$-norm of $H$ is sufficiently small, we also guarantee (H3).

(Fixed points) By (iii), $\Phi \mid \tilde{N}$ has no fixed points. Since $\Phi=\phi$ on $\Sigma \backslash$ $\left(\Sigma_{0} \cup \tilde{N}\right)$, we therefore have

$$
\operatorname{Fix}(\Phi)=\left(\operatorname{Crit}(H) \cap \Sigma_{0}\right) \cup\left(\operatorname{Fix}(\phi) \backslash \Sigma_{0}\right) .
$$

In particular, $\Phi$ only has non-degenerate fixed points and the $\mathbb{Z}_{2}$-degree of a fixed point is given by

$$
\operatorname{deg}(y)=\operatorname{ind}_{H}(y) \bmod 2 \quad \forall y \in \operatorname{Crit}(H) \cap \Sigma_{0},
$$

and

$$
\operatorname{deg}(y)=0 \bmod 2 \quad \forall y \in \operatorname{Fix}(\phi) \backslash \Sigma_{0} .
$$

The first equality follows from [23, Lemma 7.2], the second from Proposition 9. Moreover, Proposition 9 implies that every $y \in \operatorname{Fix}(\phi) \backslash \Sigma_{0}$ forms a different fixed point class of $\Phi$. This has an immediate consequence for the Floer complex $\left(C F_{*}(\Phi), \partial_{J}\right)$ with respect to a generic $J=\left(J_{t}\right)_{t \in \mathbb{R}}$.

Lemma 16. $\left(C F_{*}(\Phi), \partial_{J}\right)$ splits into the subcomplexes $\left(\mathcal{C}_{1}, \partial_{1}\right)$ and $\left(\mathcal{C}_{2}, \partial_{2}\right)$, where $\mathcal{C}_{1}$ is generated by $\operatorname{Crit}(H) \cap \Sigma_{0}$ and $\mathcal{C}_{2}$ by $\operatorname{Fix}(\phi) \backslash \Sigma_{0}$. Moreover, $\mathcal{C}_{2}$ is graded by 0 and $\partial_{2}=0$. The splitting is respected by the quantum cap action (4). 
Proof. If $y^{ \pm} \in \operatorname{Fix}(\Phi)$ are in different fixed point classes, then

$$
\mathcal{M}\left(y^{-}, y^{+} ; J, \phi\right)=\emptyset .
$$

This follows from the first equation in (2) and proves the lemma.

Next we show that hypothesis (H4) holds.

Lemma 17 (Action 2). If $\omega^{\prime}$ is a $\Phi$-invariant area form such that $\omega^{\prime}=\omega$ on $\Sigma \backslash N_{0}$, then $\alpha_{\omega^{\prime}}$ has vanishing periods.

Proof. The proof is an extension of the proof of Proposition 13. Let $u$ be a loop in $\Omega_{\Phi}$. We claim that $\left\langle\left[\alpha_{\omega^{\prime}}\right],[u]\right\rangle=0$. Since $\Phi^{\ell}$ is isotopic to $\phi^{\ell}$, we can assume that $u(., 0)$ is contained either in $\Sigma \backslash\left(\Sigma_{0} \cup \tilde{N}\right)$ or in $\Sigma_{0} \backslash N_{0}$. The argument is similar as in the above mentioned proof and relies on Lemma 12 and Corollary 21. The first case reduces to the case considered in Proposition 13. In the second case, define $h: S^{1} \times \mathbb{R} \rightarrow \Sigma_{0} \backslash N_{0}$ by $h(s, t):=\psi_{-t}(u(s, 0)) ; h$ is a loop in $\Omega_{\Phi}$. Since $\omega^{\prime}=\omega$ on $\operatorname{im}(h)$, it follows that $\int h^{*} \omega^{\prime}=\int h^{*} \omega=0$ and hence, from Lemma 12 that $\int u^{*} \omega^{\prime}=0$.

(Path of complex structures) Let $J_{0}$ be a $\omega$-compatible complex structure on $\Sigma$ which restricts to the standard complex structure on $N_{0}$. Let $J=\left(J_{t}\right)_{t \in \mathbb{R}}$ be a smooth path of $\omega$-compatible complex structures such that $J_{t+1}=\Phi^{*} J_{t}$ and $J_{t}(x)=\left(\psi_{t}^{*} J_{0}\right)(x)$ for all $t \in \mathbb{R}$ and $x \in \Sigma_{0}$. The existence of such a $J$ relies on the contractibility of the space of $\omega$ compatible complex structures on $\Sigma$. Note that $J$ satisfies (H5). Below, we impose an additional regularity condition on $J_{0}$.

We are now in position to apply Proposition 14 and compute the homology of $\left(\mathcal{C}_{1}, \partial_{1}\right)$. Let $\partial_{ \pm} \Sigma_{0}$ denote the union of components of $\partial \Sigma_{0}$ where in a neighborhood, $\Phi$ is given by $(q, p) \mapsto(q, p \mp \delta)$.

Lemma 18. The homology of $\left(\mathcal{C}_{1}, \partial_{1}\right)$ is isomorphic to $H_{*}\left(\Sigma_{0}, \partial_{+} \Sigma_{0} ; \mathbb{Z}_{2}\right)$. The quantum cap product is given by the ordinary cap product

$$
H^{*}\left(\Sigma ; \mathbb{Z}_{2}\right) \otimes H_{*}\left(\Sigma_{0}, \partial_{+} \Sigma_{0} ; \mathbb{Z}_{2}\right) \longrightarrow H_{*}\left(\Sigma_{0}, \partial_{+} \Sigma_{0} ; \mathbb{Z}_{2}\right)
$$

Proof. The proof is by the same technique as in [26]. By modifying $J_{0}$ in a neighborhood of $\operatorname{Crit}(H) \cap \Sigma_{0}$, we can assume that $\nabla H$ is a Morse-Smale vector field on $\Sigma_{0}$, where the gradient is with respect to the metric $\omega\left(., J_{0}\right.$.). This means that stable and unstable manifolds are all transverse to each other and that the stable, unstable manifolds are all transverse to $\partial \Sigma_{0}$.

Note that by Proposition $9,\left(\mathcal{C}_{1}, \partial_{1}\right)$ splits into subcomplexes generated by fixed points of $\Phi$ which are in the same connected component of 
$\Sigma_{0}$. Let $x^{ \pm}$be a pair of such fixed points and set $\mathcal{M}:=\mathcal{M}\left(x^{-}, x^{+} ; J, \Phi\right)$. For every $u \in \mathcal{M}$, it follows from Proposition 14 that $\operatorname{im}(u) \subset \Sigma_{0}$. Define the map $\tilde{u}: \mathbb{R}^{2} \rightarrow \Sigma_{0},(s, t) \mapsto \psi_{t}(u(s, t))$. A straight forward calculation, using that $u(s, t)=\psi_{1}(u(s, t+1)), \psi_{t} \circ \psi_{1}=\psi_{t+1}$ and $J_{t}=\psi_{t}^{*} J_{0}$ on $\operatorname{im}(u)$, shows that

$$
\left\{\begin{array}{l}
\tilde{u}(s, t)=\tilde{u}(s, t+1) \\
\partial_{s} \tilde{u}+J_{0}(\tilde{u})\left(\partial_{t} \tilde{u}-X_{H}(\tilde{u})\right)=0 \\
\lim _{s \rightarrow \pm \infty} \tilde{u}(s, t)=x^{ \pm}
\end{array}\right.
$$

where $X_{H}$ denotes the Hamiltonian vector field of $H$. The system (10) was studied in [23, Theorem 7.3]. There it is shown that if $H \mid \Sigma_{0}$ is replaced by $\varepsilon \cdot H \mid \Sigma_{0}$ with $\varepsilon>0$ sufficiently small, then every solution of (10) is independent of the $t$-variable and is therefore a solution of

$$
\mathrm{d} \tilde{u} / \mathrm{d} s=\varepsilon \cdot \nabla H(\tilde{u}) .
$$

Moreover, $\tilde{u}$ is regular in the sense that the operator which is defined by linearizing the equations (10) is surjective, see page 721 . If we go back to the definition of $H$, we can assume from now on that $\varepsilon=1$. That the ambient space $\Sigma_{0}$ has non-empty boundary does not affect the argument in [23, Theorem 7.3]. It is essential however that $\pi_{2}\left(\Sigma_{0}\right)=0$.

It follows that every $u \in \mathcal{M}$ is regular and that the map $u \mapsto$ $\tilde{u}$ induces a diffeomorphism of $\mathcal{M}$ and the space of (parameterized) flow lines of $\nabla H$ which are contained in $\Sigma_{0}$ and connect the critical points $x^{ \pm}$. Furthermore, these diffeomorphisms, one for each pair $x^{ \pm}$, induce an isomorphism $\left(\mathcal{C}_{1}, \partial_{1}\right) \cong\left(C M_{*}\left(H \mid \Sigma_{0}\right), \partial_{\nabla H}\right)$ of chain complexes. Here, $C M_{*}\left(H \mid \Sigma_{0}\right)$ is freely generated by $\operatorname{Crit}(H) \cap \Sigma_{0}$ and $\partial_{\nabla H}$ is defined by counting index-1 flow lines of $\nabla H$ which are contained in $\Sigma_{0}$. Note that $\nabla H$ points outwards/inwards at a component of $\partial_{+} / \partial_{-} \Sigma_{0}$. The homology of $\left(C M_{*}\left(H \mid \Sigma_{0}\right), \partial_{\nabla H}\right)$ is therefore isomorphic to $H_{*}\left(\Sigma_{0}, \partial_{+} \Sigma_{0} ; \mathbb{Z}_{2}\right)$. See $[\mathbf{2 4}]$ for details on relative Morse homology.

Similarly, we can identify the quantum cap product, defined in (4). Note that the image of the evaluation map $\mathcal{M} \rightarrow \Sigma, u \mapsto u(0,0)$, is $W^{u}\left(\nabla H, x^{-}\right) \cap W^{s}\left(\nabla H, x^{+}\right)$. Choose a Morse function $f: \Sigma \rightarrow \mathbb{R}$ such that the evaluation map is transverse to $W^{u}(\nabla f, x)$ for all $x \in$ Crit $(f)$. For $x \in \operatorname{Crit}(f)$ and $x^{ \pm} \in \operatorname{Crit}(H) \cap \Sigma_{0}$ with $\operatorname{ind}_{H}\left(x^{+}\right)=$ $\operatorname{ind}_{H}\left(x^{-}\right)+\operatorname{ind}_{f}(x)$, let $q\left(x ; x^{-}, x^{+}\right) \in \mathbb{Z}_{2}$ be the cardinality mod 2 of $W^{u}(\nabla f, x) \cap W^{u}\left(\nabla H, x^{-}\right) \cap W^{s}\left(\nabla H, x^{+}\right)$. The map

$$
C M^{*}(f) \otimes C M_{*}\left(H \mid \Sigma_{0}\right) \longrightarrow C M_{*}\left(H \mid \Sigma_{0}\right), \quad x \otimes y \longmapsto \sum_{z} q(x ; y, z) z,
$$


which induces the quantum cap product on homology, is therefore given in purely Morse theoretical terms. On the level of homology, it is the ordinary cap product. This finishes the proof of the lemma.

Proof of Theorem 1. From Lemma 16 and 18, it follows that

$$
H F_{*}(\phi) \cong H_{*}\left(\Sigma_{0}, \partial_{+} \Sigma_{0} ; \mathbb{Z}_{2}\right) \oplus \mathbb{Z}_{2}^{\# \operatorname{Fix}\left(\phi|\Sigma| \Sigma_{0}\right)}
$$

Moreover, $H^{*}\left(\Sigma ; \mathbb{Z}_{2}\right)$ acts on the first summand by ordinary cap product. Since every fixed point of $\phi \mid \Sigma \backslash \Sigma_{0}$ has fixed point index 1, the Lefschetz fixed point formula implies that

$$
\#\left(\operatorname{Fix}(\phi) \backslash \Sigma_{0}\right)=\Lambda\left(\phi \mid \Sigma \backslash \Sigma_{0}\right)
$$

It remains to show that $1 \in H^{0}\left(\Sigma ; \mathbb{Z}_{2}\right)$ acts on $\mathbb{Z}_{2}^{\Lambda\left(\phi|\Sigma| \Sigma_{0}\right)}$ by the identity and any element of $H^{1}\left(\Sigma ; \mathbb{Z}_{2}\right) \oplus H^{2}\left(\Sigma ; \mathbb{Z}_{2}\right)$ by the zero map.

From the proof of Lemma 16, we know that if $\mathcal{M}\left(y^{-}, y^{+} ; J, \Phi\right) \neq \emptyset$ for some $y^{-}, y^{+} \in \operatorname{Fix}(\phi) \backslash \Sigma_{0}$, then $y^{-}=y^{+}$. Since the action $\alpha_{\omega}$ has vanishing periods on $\Omega_{\Phi}$, by Lemma 17 , it follows that every $u \in$ $\mathcal{M}(y, y ; J, \Phi)$ has energy zero. Hence, $\mathcal{M}(y, y ; J, \Phi)=\mathcal{M}_{0}(y, y ; J, \Phi)$ consists of the constant map. Now choose a Morse function $f: \Sigma \rightarrow \mathbb{R}$ with only one critical point $x_{0}$ of index 0 and such that $\operatorname{Fix}\left(\phi \mid \Sigma \backslash \Sigma_{0}\right) \subset$ $W^{u}\left(\nabla f, x_{0}\right)$. It follows that $q\left(x ; y^{-}, y^{+}\right) \neq 0$ if and only if $x=x_{0}$ and $y^{-}=y^{+}$. This ends the proof.

Remark. Using Theorem 1, we can verify Seidel's result [28, Theorem 1] in the special case of an algebraically finite mapping class: if $g$ is a non-trivial mapping class, then the quantum cap product is trivial on $H^{2}\left(\Sigma ; \mathbb{Z}_{2}\right) \otimes H F_{*}(g)$.

To see this, assume that $g$ is algebraically finite. By Theorem 1, the only possibly non-trivial part of the the quantum cap product is given by the cap product on $H^{*}\left(\Sigma ; \mathbb{Z}_{2}\right) \otimes H_{*}\left(\Sigma_{0}, \partial_{+} \Sigma_{0} ; \mathbb{Z}_{2}\right)$. The submanifold $\Sigma_{0} \subset \Sigma$ has non-trivial boundary since $g$ is non-trivial. Now the cap product on $H^{*}\left(\Sigma ; \mathbb{Z}_{2}\right) \otimes H_{*}\left(\Sigma_{0}, \partial_{+} \Sigma_{0} ; \mathbb{Z}_{2}\right)$ factors through the homomorphism $\iota^{*}: H^{*}\left(\Sigma ; \mathbb{Z}_{2}\right) \rightarrow H^{*}\left(\Sigma_{0} ; \mathbb{Z}_{2}\right)$ which is induced by the inclusion $\iota: \Sigma_{0} \hookrightarrow \Sigma$. Since $H^{2}\left(\Sigma_{0} ; \mathbb{Z}_{2}\right)=0$, this proves the claim.

Similarly, it follows from Theorem 1 that if $\alpha \in H^{1}\left(\Sigma ; \mathbb{Z}_{2}\right)$ acts nontrivially on $H F_{*}(g)$, then there exists a map $\gamma: S^{1} \rightarrow \Sigma_{0}$ such that $\langle\alpha,[\gamma]\rangle=1$. This is a special case of $[28$, Theorem 2]. 


\section{Isolated plane curve singularities}

In this section we prove Theorem 2 and Corollary 3. We begin with a brief summary of the basic facts on isolated plane curve singularities. The standard reference is Milnor's book [18].

An isolated plane curve singularity is a germ $[f]$ of holomorphic functions $f:(U, 0) \rightarrow(\mathbb{C}, 0)$, where $U \subset \mathbb{C}^{2}$ is a neighborhood of 0 , with $(\mathrm{d} f)^{-1}(0)=\{0\}$. Let $f: U \rightarrow \mathbb{C}$ be such a function. For $\varepsilon>0$ sufficiently small, the singular fiber $f^{-1}(0)$ intersects transversally with the 3 -sphere $S_{\varepsilon}:=\left\{(x, y) \in \mathbb{C}^{2}:|x|^{2}+|y|^{2}=\varepsilon\right\}$. The intersection $L:=f^{-1}(0) \cap S_{\varepsilon} \subset S_{\varepsilon}$ is a compact oriented 1-manifold, i.e. a link. A link obtained in this way is called an algebraic link. An algebraic link is a fibred link: the map

$$
\pi: S_{\varepsilon} \backslash L \longrightarrow\{z \in \mathbb{C}:|z|=1\}, \quad z \longmapsto f(z) /|f(z)|
$$

is a fibration, the Milnor fibration, and the Milnor fiber $M:=\pi^{-1}(1) \cup$ $L$ is a Seifert surface of $L$. This means that $M \subset S_{\varepsilon}$ is an compact connected oriented embedded 2-manifold with $\partial M=L$.

The geometric monodromy is an isotopy class of the group $\operatorname{Diff}_{c}^{+}(M)$ of orientation preserving diffeomorphisms which are the identity near $\partial M$ and is defined as follows. Given a connection on $S_{\varepsilon} \backslash L$, i.e. a rank1 subbundle of the tangent bundle of $S_{\varepsilon} \backslash L$ which is transversal to $\operatorname{ker}(\mathrm{d} \pi)$, parallel transport induces an orientation preserving diffeomorphism of $\pi^{-1}(1)$; a so-called characteristic diffeomorphism. To extend this diffeomorphism to $M$, we specify the connection in a neighborhood of $L$. For this observe the following.

Let $L^{\prime}$ be a connected component of $L$ and $T \subset S_{\varepsilon}$ be a tubular neighborhood of $L^{\prime}$. A standard meridian of $\left(T, L^{\prime}\right)$ is an embedded circle in $T \backslash L^{\prime}$, which is homologically trivial in $T$ and has linking number 1 with $L^{\prime}$ in $S_{\varepsilon}$. There is a fibration of $T \backslash L^{\prime}$ such that every fiber is a standard meridian of $\left(T, L^{\prime}\right)$. This fibration is unique up to isotopy and if $T$ is sufficiently small, induces a connection on $T \backslash L^{\prime}$. In this way, we get a standard connection in neighborhood of $L$. A connection on $S_{\varepsilon} \backslash L$ which restricts to this standard connection induces a characteristic diffeomorphism which is compactly supported and hence extends trivially to $M$. Moreover, any two such diffeomorphisms are isotopic in $\operatorname{Diff}_{c}^{+}(M)$. The isotopy class obtained in this way is therefore an invariant of $\pi$.

The proof of Theorem 2 relies on the following result, which is a refinement of the classical result of A'Campo [2] and Lê [17] that the 
Lefschetz number of the geometric monodromy of an isolated plane curve singularity vanishes. We use the following notation: we denote by $\iota: \operatorname{Diff}_{c}^{+}(M) \rightarrow \operatorname{Diff}^{+}(M, \partial M)$ the inclusion, where $\operatorname{Diff}^{+}(M, \partial M)$ denotes the group of orientation preserving diffeomorphisms which are the identity on $\partial M$.

Proposition 19. Let $M$ be the Milnor fiber and $g$ be the geometric monodromy of an isolated plane curve singularity. There is a representative $\phi \in \iota_{*} g$ which is a diffeomorphism of finite type (same definition as for closed surfaces) and such that $\operatorname{Fix}(\phi)=\partial M$. Moreover, $\phi$ only has positive twists.

As already mentioned in the introduction, this proposition follows from the work of A'Campo [1], [4] on the geometric monodromy. Our proof, given in Appendix B, relies on the work of Eisenbud and Neumann [10] on the monodromy of plane curve singularities. The relevant results from [10] are summarized in Appendix B. We remark that the vanishing of the Lefschetz number of diffeomorphism of finite type is not enough to exclude the existence of pointwise fixed annuli.

Proof of Theorem 2. We recall that $\Sigma$ denotes a closed oriented 2-manifold of genus $\geq 2$ and $M \subset \Sigma$ the Milnor fiber of an isolated plane curve singularity. Moreover, $g$ denotes the mapping class of $\Sigma$ which is obtained by extending the geometric monodromy of the singularity trivially to $\Sigma$.

At this point we distinguish two cases. Recall that the twist map components of a finite type diffeomorphism are supported on noncontractible annuli. Hence, we first assume that no component of $\Sigma \backslash$ $\operatorname{int}(M)$ is a disk. From Proposition 19, it follows that there exists a representative $\phi \in g$ which is of finite type and such that $\Sigma_{0}=\Sigma \backslash \operatorname{int}(M)$, $\partial_{+} \Sigma_{0}=\partial M$ and $\Lambda(\phi \mid \operatorname{int}(M))=0$. Theorem 1 therefore implies that

$$
H F_{*}(\phi) \cong H_{*}\left(\Sigma \backslash \operatorname{int}(M), \partial M ; \mathbb{Z}_{2}\right) .
$$

By excision, it follows that $H F_{*}(\phi) \cong H_{*}\left(\Sigma, M ; \mathbb{Z}_{2}\right)$.

Now assume that $D_{1}, \ldots, D_{n}$ are disk components of $\Sigma \backslash \operatorname{int}(M)$. Set $M^{\prime}:=M \cup D_{1} \cup \cdots \cup D_{n}$. We claim that there exists a representative $\phi \in g$ which is of finite type and such that $\Sigma_{0}=\Sigma \backslash \operatorname{int}\left(M^{\prime}\right), \partial_{+} \Sigma_{0}=$ $\partial M^{\prime}$ and $\Lambda\left(\phi \mid \operatorname{int}\left(M^{\prime}\right)\right)=n$. Theorem 1 then implies that

$$
H F_{*}(\phi) \cong H_{*}\left(\Sigma \backslash \operatorname{int}\left(M^{\prime}\right), \partial M^{\prime} ; \mathbb{Z}_{2}\right) \oplus \mathbb{Z}_{2}^{n} \cong H_{*}\left(\Sigma, M^{\prime} ; \mathbb{Z}_{2}\right) \oplus \mathbb{Z}_{2}^{n} .
$$


Since $H_{*}\left(\Sigma, M^{\prime} ; \mathbb{Z}_{2}\right) \oplus \mathbb{Z}_{2}^{n} \cong H_{*}\left(\Sigma, M ; \mathbb{Z}_{2}\right)$, this proves Theorem 2 up to the claim above. The claim follows from Proposition 19 by "collapsing" each disk component of $\Sigma \backslash \operatorname{int}(M)$ to a point.

Next we prove Corollary 3. First, we recall some terminology. Let $k>0$; an $A_{k}$-configuration in $\Sigma$ is a $k$-tuple $\left(C_{1}, \ldots, C_{k}\right)$ of embedded circles in $\Sigma$ such that

$\#\left(C_{i} \pitchfork C_{i+1}\right)=1 \quad$ for $i=1, \ldots, k-1, \quad \#\left(C_{i} \pitchfork C_{j}\right)=0 \quad$ if $|i-j|>1$.

The $A_{k}$-singularity is the germ of the function $f(x, y)=x^{2}+y^{k+1}$ at $(0,0)$. It is a classical result in the theory of singularities, that the Milnor fiber $M$ of this singularity contains an $A_{k}$-configuration, which is (i) a spine of $M$ and (ii) a distinguished basis of vanishing cycles. See [5, Section 2.9], [3].

Proof of Corollary 3. Let $\left(C_{1}, \ldots, C_{k}\right)$ be an $A_{k}$-configuration in $\Sigma$. From remark (i) above, it follows that a tubular neighborhood $N$ of $C_{1} \cup \cdots \cup C_{k}$ can be identified with the Milnor fiber of the $A_{k}$-singularity such that, by (ii), $C_{1} \cup \cdots \cup C_{k}$ is a distinguished set of vanishing cycles. This implies that the class $g$ of the product $\tau_{1} \circ \cdots \circ \tau_{k}$ of right Dehn twists can also be obtained by extending the geometric monodromy of the $A_{k}$-singularity trivially to $\Sigma$. It therefore follows from Theorem 2 that

$$
H F_{*}(g) \cong H_{*}\left(\Sigma, N ; \mathbb{Z}_{2}\right) \cong H_{*}\left(\Sigma, C_{1} \cup \cdots \cup C_{k} ; \mathbb{Z}_{2}\right) .
$$

This together with naturality of Floer homology proves the corollary.

Proof of Theorem 4. Let $M$ be the Milnor fiber and $g$ be the geometric monodromy of an isolated plane curve singularity. Let $\phi \in \iota_{*} g$ be as in Proposition 19. Since $\phi$ only has positive twists, we can perturb it near the boundary to a diffeomorphism $\phi_{+}$such that $\operatorname{Fix}\left(\phi_{+}\right)=\emptyset$. Furthermore, it follows as in the proof of Proposition 10, that if $\omega$ is a $\phi$-invariant area form on $M$, then $\left[\omega_{\phi}\right]=0$. Hence, $\phi$ is monotone in the sense defined in Appendix C. Therefore, $H F_{*}(g,+)=H F_{*}(\phi,+)=$ 0 .

\section{Appendix A. Products of disjoint Dehn twists}

The goal of this appendix is to prove Proposition 20, which was also stated in [26, Lemma 3]. This result is used for the proof of the fixed point as well as the action proposition in Section 3. Let $I$ denote either 
$[0,1]$ or $S^{1}$. For every continuous map $\gamma: I \rightarrow \Sigma$ and compact 1dimensional submanifold $C \subset \Sigma$, there is the geometric intersection number

$$
i(\gamma, C)=\min \left\{\# \beta^{-1}(C) \mid \beta \text { is homotopic rel endpoints to } \gamma\right\} .
$$

If $I=S^{1}$, homotopic rel endpoints means freely homotopic.

Proposition 20. Let $C \subset \Sigma$ be a finite union of non-contractible disjoint circles. Let $\phi$ be a product of Dehn twists along $C$ which twists with the same sign along parallel components of $C$. Let $\gamma: I \rightarrow \Sigma$ be such that $\gamma(\partial I) \cap \operatorname{supp}(\phi)=\emptyset$. If $\gamma$ is homotopic rel endpoints to $\phi \circ \gamma$, then $i(\gamma, C)=0$.

An immediate consequence is the following

Corollary 21. Let $\phi$ be of finite type and $\ell>0$ be as such that $\phi^{\ell}|\Sigma|$ $N=$ id. Let $\gamma: I \rightarrow \Sigma$ be such that $\gamma(\partial I) \cap \operatorname{supp}\left(\phi^{\ell}\right)=\emptyset$. If $\gamma$ is homotopic rel endpoints to $\phi^{\ell} \circ \gamma$, then there exists $\gamma^{\prime}: I \rightarrow \operatorname{Fix}\left(\phi^{\ell}\right)$ which is homotopic rel endpoints to $\gamma$.

Proof of Proposition 20. We begin with the case $I=[0,1]$. For every component of $C$, let $N^{\prime}$ be the closed tubular neighborhood, where the Dehn twist is supported. For different components of $C$, these tubular neighborhoods are disjoint. Let $N$ be the union of the $N^{\prime}$ and $\tilde{C}:=$ $\partial N$. We prove in several steps that $i(\gamma, \tilde{C})=0$ if $\gamma$ is homotopic rel endpoints to $\phi \circ \gamma$.

Let $\beta:[0,1] \rightarrow \Sigma$ and $u:[0,1]^{2} \rightarrow \Sigma$ be such that $\# \beta^{-1}(\tilde{C})=i(\gamma, \tilde{C})$ and

$$
u(s, 0)=\beta(s), \quad u(s, 1)=\phi(\beta(s)), \quad u(0, t)=\beta(0), \quad u(1, t)=\beta(1)
$$

for all $s, t \in[0,1]$. We assume that $\beta(0), \beta(1) \in \Sigma \backslash N$. Without loss of generality we further assume that $u$ is transverse to $\tilde{C}$. Hence $B:=u^{-1}(\tilde{C}) \subset[0,1]^{2}$ is a compact 1-dimensional submanifold with boundary $\partial B=\beta^{-1}(\tilde{C}) \cup(\phi \circ \beta)^{-1}(\tilde{C})$. Every component of $B$ is either a circle or an arc.

Claim 1. We may assume that no component of $B$ is a circle.

Let $S$ be a circle component of $B$. The interior of $S$, i.e. the region of $[0,1]^{2}$ bounded by $S$, is a disk. The restriction of $u$ to this disk induces an element of $\pi_{2}\left(\Sigma, C^{\prime}\right)$, where $C^{\prime}$ is the component of $\tilde{C}$ where $S$ is mapped to. Since $C^{\prime}$ is non-contractible, $\pi_{2}\left(\Sigma, C^{\prime}\right)=0$, and hence $u$ can be deformed in a neighborhood of the interior of $S$ in such a way that $B$ has less circle components. Repeating this argument finitely many 
times proves claim 1 . From now on, we assume that every component of $B$ is an arc.

Claim 2. There is no component $B^{\prime}$ of $B$ with $\partial B^{\prime} \subset[0,1] \times 0$ or $\partial B^{\prime} \subset[0,1] \times 1$.

Assume that $B^{\prime}$ is such that $\partial B^{\prime} \subset[0,1] \times 0$. Note that the boundary points of $B^{\prime}$ are intersection points of $\beta$ with $\tilde{C}$. Since $B^{\prime}$ is mapped to some component of $\tilde{C}$ under $u$, it follows that these intersection points can be removed by a homotopy of $\beta$. This however, contradicts the definition of $\beta$. Hence every $B^{\prime}$ with one boundary point on $[0,1] \times 0$ has the other boundary point on $[0,1] \times 1$. On the other hand, $B$ has the same the number of boundary points on $[0,1] \times 0$ as on $[0,1] \times 1$, since $\beta$ and $\phi \circ \beta$ intersect $\tilde{C}$ in the same number of points. This proves claim 2. The rest of the proof is devoted to

Claim 3. $B$ is empty, i.e. $\#(\operatorname{im} \beta \cap \tilde{C})=0$.

The proof is by contradiction; assume that $B \neq \emptyset$. First we define an integer valued function on $\pi_{0}(B)$. Let $B^{\prime}$ be a component of $B$. By claim 1 and $2, B^{\prime}$ is an arc and $\partial B^{\prime}=\{(b, 0),(b, 1)\}$, for some $b \in(0,1)$. Let $C^{\prime}$ be the component of $\tilde{C}$ where $B^{\prime}$ is mapped to under $u$. Since $u(b, 1)=\phi(u(b, 0))=u(b, 0)$, the restriction $u \mid B^{\prime}$ has a well defined mapping degree $\operatorname{deg} u \mid B^{\prime}$, once orientations of $B^{\prime}$ and $C^{\prime}$ are fixed. We orient $B^{\prime}$ "from the bottom to the top" and choose an orientation of $\tilde{C}$ such that the orientations of two homotopic components match. We thus have the map

$$
\mathrm{d}: \pi_{0}(B) \longrightarrow \mathbb{Z}, \quad B^{\prime} \longmapsto \operatorname{deg} u \mid B^{\prime} .
$$

Let $\pi_{0}(B)=\left\{B_{1}, \ldots, B_{2 n}\right\}$ be ordered such that

$$
b_{i}<b_{j} \Longleftrightarrow i<j,
$$

where $\partial B_{i}=\left\{\left(b_{i}, 0\right),\left(b_{i}, 1\right)\right\}$. Note that the cardinality of $\pi_{0}(B)$ is even, since $\beta(0)$ and $\beta(1)$ are both in the complement of $N$. Observe that the loops $u \mid B_{1}$ and $u \mid B_{2 n}$ are both homotopic to the constant loop and hence

$$
\mathrm{d}\left(B_{1}\right)=\mathrm{d}\left(B_{2 n}\right)=0 .
$$

To prove claim 3 , we will now show that $\mathrm{d}\left(B_{2 n}\right) \neq 0$, which is a contradiction. In fact, we will prove by induction that

$\left(\star_{k}\right) \quad \mathrm{d}\left(B_{2 k}\right) \neq 0, \quad \operatorname{sign}\left(b_{2 k}\right)=\operatorname{sign}\left(b_{2 k-2}\right) \quad$ and $\quad C_{2 k} \sim C_{2 k-2}$,

for all $1 \leq k \leq n$. Here $\operatorname{sign}\left(b_{i}\right)$ denotes the sign of $b_{i}$ as an intersection point of $\beta$ and $\tilde{C}$ and $C_{i}$ is the component of $\tilde{C}$ where $B_{i}$ is mapped 
to under $u$. We will use the following formula for the function $\mathrm{d}$, which we prove later on:

$$
\mathrm{d}\left(B_{2 k}\right)=-\sum_{i=1}^{k} \operatorname{sign}\left(b_{2 i}\right) \cdot \varepsilon_{2 i},
$$

for all $1 \leq k \leq \ell$. Here $\varepsilon_{i}= \pm$ is the sign of the twist of $\phi$ along $C_{i}$. For $k=1$, formula (11) gives $\mathrm{d}\left(B_{2}\right)=\operatorname{sign}\left(b_{2}\right) \cdot \varepsilon_{2} \neq 0$. This proves the induction hypothesis $\left(\star_{1}\right)$, since the other two statements are empty in this case.

Assume that $\left(\star_{k}\right)$ holds for all $1 \leq i \leq k<n$. To show that it also holds for $k+1$ first note that if

$$
\operatorname{sign}\left(b_{2 k}\right)=\operatorname{sign}\left(b_{2 k+2}\right) \quad \text { and } \quad C_{2 k} \sim C_{2 k+2},
$$

then by formula (11), we get that

$$
\mathrm{d}\left(B_{2 k+2}\right)=(k+1) \cdot \operatorname{sign}\left(b_{2 k+2}\right) \cdot \varepsilon_{2 k+2} \neq 0 .
$$

To prove (12), we consider the restriction of $u$ to the region $D \subset[0,1]^{2}$ that is bounded by the $\operatorname{arcs} B_{2 k}, B_{2 k+1}$ and $\left[b_{2 k}, b_{2 k+1}\right] \times 0,\left[b_{2 k}, b_{2 k+1}\right] \times 1$. Note that since $u(D) \subset \Sigma \backslash \operatorname{int}(N), u(s, 1)=u(s, 0)$ for all $s \in$ $\left[b_{2 k}, b_{2 k+1}\right]$. Hence $u \mid D$ gives a homotopy between the loops $\mathrm{d}\left(B_{2 k}\right) \cdot C_{2 k}$ and $\mathrm{d}\left(B_{2 k+1}\right) \cdot C_{2 k+1}$. This implies that $C_{2 k} \sim C_{2 k+1}$ and that $\mathrm{d}\left(B_{2 k}\right)=$ $\mathrm{d}\left(B_{2 k+1}\right)$. Since $C_{2 k+1}$ and $C_{2 k+2}$ are both contained in the boundary of one component of $N$, they are clearly homotopic and hence $C_{2 k} \sim C_{2 k+2}$. It thus remains to show that $\operatorname{sign}\left(b_{2 k}\right)=\operatorname{sign}\left(b_{2 k+2}\right)$. For this we need

Claim 4. $C_{2 k} \neq C_{2 k+1}$ and $C_{2 k+1} \neq C_{2 k+2}$.

Assume that $C_{2 k}=C_{2 k+1}$ and consider the path $s \mapsto u(s, 0), s \in$ $\left[b_{2 k}, b_{2 k+1}\right]$. We claim that since $\mathrm{d}\left(B_{2 k}\right)=\mathrm{d}\left(B_{2 k+1}\right) \neq 0$, the path can be deformed into $C_{2 k}$, which contradicts minimality of $\beta$. For the proof of the claim, we refer to [13, Corollary 5]. Similarly, $C_{2 k+1}=C_{2 k+2}$ is also a contradiction to minimality of $\beta$.

Claim 5. $\operatorname{sign}\left(b_{2 k}\right)=\operatorname{sign}\left(b_{2 k+1}\right)=\operatorname{sign}\left(b_{2 k+2}\right)$.

Since $C_{2 k}$ and $C_{2 k+1}$ are homotopic and disjoint, there exists an embedded annulus $A \subset \Sigma$ which bounds $C_{2 k}$ and $C_{2 k+1}$. Since $\mathrm{d}\left(B_{2 k}\right)=$ $\mathrm{d}\left(B_{2 k+1}\right) \neq 0$, it follows that $u(D)=A$, where $D$ is as above. Otherwise, we could find a map from the torus to $\Sigma$ with nonzero degree. Such a map does not exist since the genus of $\Sigma$ is $>1$. Thus we know that the path $\beta$ enters $A$ at $b_{2 k}$ and leaves at $b_{2 k+1}$. The intersection points therefore have the same $\operatorname{sign}, \operatorname{sign}\left(b_{2 k}\right)=\operatorname{sign}\left(b_{2 k+1}\right)$. 
The proof that $\operatorname{sign}\left(b_{2 k+1}\right)=\operatorname{sign}\left(b_{2 k+2}\right)$ is similar. In this case however, we know that the region $D^{\prime}$ of $[0,1]^{2}$ between $B_{2 k+1}$ and $B_{2 k+2}$ is mapped to a component $N^{\prime}$ of $N$ under $u$. Since $\mathrm{d}\left(B_{2 k+1}\right) \neq 0$, it follows that $u\left(D^{\prime}\right)=N^{\prime}$ and hence that $b_{2 k+1}$ and $b_{2 k+2}$ have the same sign, as above. This proves claim 5 and ends the proof of (12). It remains to proof formula (11).

Formula (11) is the consequence the following properties of the function d. First of all, as was shown above, we have that

$$
\mathrm{d}\left(B_{2 k}\right)=\mathrm{d}\left(B_{2 k+1}\right),
$$

for all $1 \leq k \leq n-1$. We now show that

$$
\mathrm{d}\left(B_{2 k}\right)=\mathrm{d}\left(B_{2 k-1}\right)-\operatorname{sign}\left(b_{2 k}\right) \cdot \varepsilon_{2 k},
$$

for all $2 \leq k \leq \ell$. The idea is to look again at the region $D^{\prime} \subset[0,1]^{2}$ which is bounded by the arcs $B_{2 k-1}, B_{2 k}$ and $\left[b_{2 k-1}, b_{2 k}\right] \times 0,\left[b_{2 k-1}, b_{2 k}\right] \times$ 1. Let $N^{\prime}$ be the component of $N$ where $D^{\prime}$ is mapped to. As in claim 4, we conclude that $\partial N^{\prime}=C_{2 k-1} \cup C_{2 k}$. Choose an orientation preserving diffeomorphism $[0,1] \times S^{1} \cong N^{\prime}$ such that $C_{2 k-1} \cong 0 \times S^{1}$ and $C_{2 k} \cong$ $1 \times S^{1}$. Assume for the moment that the orientations of $C_{2 k-1}$ and $C_{2 k}$ are compatible with these diffeomorphisms. This is equivalent to saying that $\operatorname{sign}\left(b_{2 k-1}\right)=1$. Let $\mathrm{pr}:[0,1] \times S^{1} \rightarrow S^{1}$ denote the projection onto the second factor. The map prou $: \partial D \rightarrow S^{1}$ is the composition of four loops, denoted by $v, u \mid B_{2 k}$, the inverse of $w$ and the inverse of $u \mid B_{2 k-1}$. Here $v, w$ are the loops $s \mapsto \operatorname{pr}(u(s, 0)), s \mapsto \operatorname{pr}(u(s, 1))$ for $s \in\left[b_{2 k-1}, b_{2 k}\right]$. Since the mapping degree of pr $\circ u \mid \partial D$ vanishes, it follows that

$$
\operatorname{deg} v+\mathrm{d}\left(B_{2 k}\right)-\operatorname{deg} w-\mathrm{d}\left(B_{2 k-1}\right)=0 .
$$

However, $\operatorname{deg} v-\operatorname{deg} w=\varepsilon_{2 k}$, and this proves equation (14) in the case that $\operatorname{sign}\left(b_{2 k}\right)=1$. If $\operatorname{sign}\left(b_{2 k}\right)=-1$, pr induces orientation reversing maps on $C_{2 k-1}$ and $C_{2 k}$. Hence, the same argument as above gives

$$
\operatorname{deg} v-\mathrm{d}\left(B_{2 k}\right)-\operatorname{deg} w+\mathrm{d}\left(B_{2 k-1}\right)=0,
$$

which again implies (14). Formula (11) is now an immediate consequence of $(13),(14)$ and the fact that $\mathrm{d}\left(B_{1}\right)=0$. This ends the proof of the proposition in the case $I=[0,1]$.

The proof in the case $I=S^{1}$ follows the same line of arguments as above. In this case, however, $B=u^{-1}(\tilde{C})$ is a subset of $S^{1} \times[0,1]$ instead if $[0,1]^{2}$. This does not affect the proofs of claims 1 and 2 above and hence, we can assume that every component $B^{\prime}$ of $B$ is an arc with $\partial B^{\prime}=\left\{(b, 0),\left(b^{\prime}, 1\right)\right\}$. Note that $b, b^{\prime} \in S^{1}$ are not necessarily equal. 
We show how the proof of claim 3 extends to this case. The idea is to consider the $\ell$-fold catenation $v: S^{1} \times[0, \ell] \rightarrow \Sigma$, defined by

$$
v(s, t):=\phi^{j}(u(s, t-j)) \text { for }(s, t) \in S^{1} \times[j, j+1], j<\ell,
$$

where $\ell>0$ is chosen such that every component $A^{\prime}$ of $A:=v^{-1}(\tilde{C})$ satisfies $\partial A^{\prime}=\{(a, 0),(a, \ell)\}$ for some $a \in S^{1}$. The rest of the argument is analogous to the above. Define the function $\mathrm{d}: \pi_{0}(A) \rightarrow \mathbb{Z}$ and prove that it satisfies

$$
\mathrm{d}\left(A_{2 k}\right)=\mathrm{d}\left(A_{1}\right)-k \cdot \ell \cdot \operatorname{sign}\left(a_{2 k}\right) \cdot \varepsilon_{2 k} .
$$

Here, the signs $\operatorname{sign}\left(a_{i}\right)$ and $\varepsilon_{i}$ are defined as above. The additional factor $\ell$ appears since $\phi^{\ell}$ twists with multiplicity $\ell$ along every component of $C$. The order on $\pi_{0}(A)$ is a cyclic order induced from the boundary points as above. Formula (15) is therefore a contradiction if $\pi_{0}(A) \neq \emptyset$. This ends the proof of the proposition.

\section{Appendix B. Decomposition of the monodromy}

In this appendix we prove Proposition 19. For this we use the theory of splice diagrams which was developed by Eisenbud and Neumann [10], [21] to compute invariants of plane curve singularities. In the following we summarize their results on the geometric monodromy. We would like to emphasis here that our discussion is far from being selfcontained. Instead, we only concentrate on the facts which are useful for our purpose. For details, proofs as well as the general picture, we refer to the excellent monograph [10]. Let us introduce some terminology.

Definition 22. We denote by $M$ a compact oriented 2-manifold with boundary.

(i) Let $\phi: M \rightarrow M$ be a diffeomorphism and $C \subset M$ be a $\phi$-invariant union of disjoint non-contractible circles. A $\phi$-component of $(M, C)$ is a a union of connected components of $M \backslash C$ which are cyclically permuted by $\phi$. The topological type of a $\phi$-component $M^{\prime}$ is the triple $(\chi, d, h)$, where $\chi$ is the Euler characteristic, $d$ the number of connected components and $h$ the number of ends of $\operatorname{int}\left(M^{\prime}\right)$.

(ii) An orientation preserving diffeomorphism $\phi: M \rightarrow M$ is called an admissible twist map if $M$ is a union of annuli and:

(1) $\phi$ cyclically permutes the connected components of $M$.

(2) If $n>0$ denotes the number of connected components of $M$, then $\phi^{n}$ is given by the local model

$$
[0,1] \times S^{1} \ni(q, p) \longmapsto(q, p-f(q)),
$$


where $f:[0,1] \rightarrow \mathbb{R}$ is monotone.

(3) There exists $q>0$ such that $\phi^{q} \mid \partial M=\mathrm{id}$.

If $\phi: M \rightarrow M$ is an admissible twist map, then its twist number is defined by

$$
\ell:=\frac{1}{q} \cdot\left(\operatorname{var}\left(\phi^{q}\right) \xi \cdot \xi\right) \in \mathbb{Q} .
$$

Here $\xi$ is a generator of $H_{*}\left(M^{\prime}, \partial M^{\prime}\right)$, with $M^{\prime}$ a connected component of $M, \operatorname{var}\left(\phi^{q}\right): H_{*}\left(M^{\prime}, \partial M^{\prime}\right) \rightarrow H_{*}\left(M^{\prime}\right)$ is the variation homomorphism of $\phi^{q}$ and the dot stands for the intersection pairing.

(iii) An admissible triple $(\phi, M, C)$ consists of an orientation preserving diffeomorphism $\phi: M \rightarrow M$ and a $\phi$-invariant finite union $C \subset M$ of disjoint non-contractible circles, such that the following holds. Let $M^{\prime}$ be a $\phi$-component of $(M, C)$ and $\chi$ denote its Euler characteristic.

(1) If $\chi=0$, then $\phi \mid \operatorname{cl}\left(M^{\prime}\right)$ is an admissible twist map.

(2) If $\chi<0$, then $\phi \mid \operatorname{cl}\left(M^{\prime}\right)$ is a periodic map.

By the period of a periodic diffeomorphism $\phi$, we mean the smallest integer $\ell>0$ such that $\phi^{\ell}=\mathrm{id}$.

(iv) Set

$$
\mathcal{T}:=\left\{(\chi, d, h ; \ell) \in \mathbb{Z}^{3} \times \mathbb{Q}: d, h>0 ; \chi \leq 0 ; \chi<0 \Rightarrow \ell \in \mathbb{N}_{>0}\right\} .
$$

Let $(\phi, M, C)$ be an admissible triple. For every $\phi$-component $M^{\prime}$ of $(M, C)$, set $t\left(M^{\prime}\right):=(\chi, d, h ; \ell) \in \mathcal{T}$, where $(\chi, d, h)$ denotes the topological type of $M^{\prime}$ and $\ell$ either the period of $\phi \mid M^{\prime}$ if $\chi<0$ or the twist number of $\phi \mid M^{\prime}$ if $\chi=0$. The characteristic set of $(\phi, M, C)$ is defined by

$$
\mathfrak{t}(\phi, M, C):=\left\{t\left(M^{\prime}\right): M^{\prime} \text { is a } \phi \text {-component of }(M, C)\right\} \subset \mathcal{T} .
$$

Recall that an isolated plane curve singularity, or simply isolated singularity, is a germ $[f]$ of holomorphic functions $f:(U, 0) \rightarrow(\mathbb{C}, 0)$, where $U \subset \mathbb{C}^{2}$ is a neighborhood of 0 , with $(\mathrm{d} f)^{-1}(0)=\{0\}$. The Milnor fiber of $[f]$ is a compact connected oriented 2-manifold $M$ with boundary. The geometric monodromy $g$ of $[f]$ is an isotopy class of $\operatorname{Diff}_{c}^{+}(M)$.

We now proceed as follows: we explain in B.1 how to associate a diagram $\Gamma$, called splice diagram, to $[f]$ and in B.2, how to associate a set $\mathfrak{t}_{\Gamma} \subset \mathcal{T}$ to such a diagram $\Gamma$. The significance of these constructions is expressed by the following result from [10], which we state precisely in Theorem 23: There exists an admissible triple $(\phi, M, C)$ 
with characteristic set $\mathfrak{t}_{\Gamma}$ and such that $\phi \in \iota_{*} g^{1}$. Moreover, we have a formula for the twist map components of $\phi$. This is used in B.3 to prove Proposition 19.

As the title of the monograph [10] indicates, Eisenbud and Neumann's point of view is that of link theory. Our discussion of splice diagrams, however, is without any reference to link theory. We simply consider them as a tool for encoding some algebraic data. This has the advantage that the reader is not assumed to be familiar with 3-manifold theory. The downside is that these diagrams seem to lack intrinsic geometric relevance and that it is not clear, how the stated results are actually proven. For this we refer to the original literature [10], [21].

B.1. Puiseux data and splice diagrams. Let $[f]$ be an isolated singularity. In the following, we define the diagram $\Gamma[f]$ as shown in [10, Appendix 1]. The construction can be divided into 4 steps.

Step 1. The first step uses the so-called Newton method for solving the equation $f(x, y)=0$ for $y$ in terms of $x$ in a neighborhood of 0 . We only state the result without going into detail and refer the reader to [6, Chapter 8.3]. Recall that a fractional power series is a pair $(P, d)$, where

$$
P(x)=\sum_{i=1}^{r} a_{i} x^{n_{i}}, r \in \mathbb{N} \cup\{\infty\}, a_{i} \in \mathbb{C}, a_{i} \neq 0, n_{i} \in \mathbb{N}_{>0}, n_{i}<n_{i+1},
$$

is a power series converging in a neighborhood of 0 , and $d$ is a positive integer which is relatively prime to the set $\left\{n_{i}: i \in \mathbb{N}, i \leq r\right\}$. Two fractional power series $(P, d),(\tilde{P}, \tilde{d})$ are called equivalent, we write $(P, d) \sim(\tilde{P}, \tilde{d})$ if $d=\tilde{d}$ and there exists $\theta \in \mathbb{C}$ such that $\theta^{d}=1$ and $\tilde{P}(x)=P(\theta \cdot x)$.

Now if $[f]$ is an isolated singularity, there is a collection of pairwise non-equivalent fractional power series $\left\{\left(P_{1}, d_{1}\right), \ldots,\left(P_{\kappa}, d_{\kappa}\right)\right\}$, called Puiseux series, such that

$$
f(x, y)=0 \quad \Longleftrightarrow \quad \exists z \in \mathbb{C}, \exists j \in\{1, \ldots, \kappa\}: x=z^{d_{j}}, y=P_{j}(z) .
$$

The Puiseux series are uniquely determined up to equivalence; $\kappa$ is the number of branches of $[f]$.

Step 2. Given a collection of pairwise non-equivalent fractional power series $\left\{\left(P_{1}, d_{1}\right), \ldots,\left(P_{\kappa}, d_{\kappa}\right)\right\}$, we now define another such collection, denoted by $\left\{\left(P_{1}^{\prime}, d_{1}\right), \ldots,\left(P_{\kappa}^{\prime}, d_{\kappa}\right)\right\}$, with the property that each $P_{j}^{\prime}$ is a

\footnotetext{
${ }^{1}$ Here, $\iota: \operatorname{Diff}_{c}^{+}(M) \rightarrow \operatorname{Diff}^{+}(M, \partial M)$ is the inclusion.
} 
finite series. For this we need the following notation. Let $\Pi=(P, d)$ be a fractional power series. For $s \in \mathbb{N}$, set

$$
d_{s}(\Pi):=\min \left\{d^{\prime} \in \mathbb{N}: 1 \leq i \leq \min (s, r) \Rightarrow d^{\prime} \cdot n_{i} \in d \cdot \mathbb{N}\right\}
$$

and

$$
P^{(s)}(x):=\sum_{i=1}^{\min (s, r)} a_{i} x^{m_{i}}, \quad m_{i}:=n_{i} d_{s}(\Pi) / d .
$$

Note that $\Pi^{(s)}:=\left(P^{(s)}, d_{s}(\Pi)\right)$ is a fractional power series and that $d_{s}(P, d)$ is increasing in $s$ and eventually equals $d$. For every $j=$ $1, \ldots, \kappa$, set $\Pi_{j}:=\left(P_{j}, d_{j}\right)$ and define

$$
r_{j}:=\min \left\{s \in \mathbb{N}: d_{s}\left(\Pi_{j}\right)=d_{j} \text { and } j \neq j^{\prime} \Rightarrow \Pi_{j}^{(s)} \not \Pi_{j^{\prime}}^{(s)}\right\} .
$$

Note that $\Pi_{j}^{\left(r_{j}\right)} \not \Pi_{i}^{\left(r_{i}\right)}$ if $j \neq i$. Now let $\left\{\Pi_{1}, \ldots, \Pi_{\kappa}\right\}$ be the Puiseux series of $[f]$. We call $\left\{\Pi_{1}^{\left(r_{1}\right)}, \ldots, \Pi_{\kappa}^{\left(r_{\kappa}\right)}\right\}$ the Puiseux data of $[f]$.

Step 3. Using the Puiseux data of $[f]$, one can now define the diagram $\tilde{\Gamma}[f]$ from which the diagram $\Gamma[f]$ is obtained in the next step. For the sake of simplicity, we give the precise definition of $\tilde{\Gamma}[f]$ only for the cases $\kappa=1,2$. Assume that $\kappa=1$ and let $(P, d)$ denote the Puiseux data. Define the integers $q_{1}, \ldots, q_{r}, p_{1}, \ldots, p_{r}>0$ such that $\operatorname{gcd}\left(q_{i}, p_{i}\right)=1$ and

$$
P\left(x^{1 / d}\right)=x^{\frac{q_{1}}{p_{1}}}\left(a_{1}+x^{\frac{q_{2}}{p_{1} p_{2}}}\left(\ldots\left(a_{r-1}+a_{r} x^{\frac{q_{r}}{p_{1} \cdots p_{r}}}\right) \ldots\right)\right) .
$$

Define the integers $\alpha_{1}, \ldots, \alpha_{r}$ recursively by

$$
\alpha_{1}=q_{1}, \quad \alpha_{i+1}=p_{i} p_{i+1} \alpha_{i}+q_{i} .
$$

Note that $\operatorname{gcd}\left(\alpha_{i}, p_{i}\right)=1$ for all $i=1, \ldots, r$. The graph $\tilde{\Gamma}[f]$ is given by

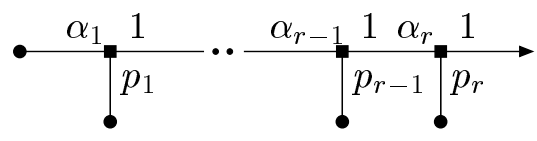

The coefficients $a_{i}$ only enter the description of $\tilde{\Gamma}[f]$ if $\kappa>1$. Let $\{\Pi, \tilde{\Pi}\}$ be the Puiseux data of $[f]$. To give the diagram $\tilde{\Gamma}[f]$ one distinguishes three cases. Set

$$
t:=\min \left\{s \geq 0: \Pi^{(s+1)} \not \tilde{\Pi}^{(s+1)}\right\}
$$

and let $\tilde{r}, \tilde{\alpha}_{1}, \ldots, \tilde{\alpha}_{\tilde{r}}, \tilde{p}_{1}, \ldots, \tilde{p}_{\tilde{r}}$ denote the integers associated to $\tilde{\Pi}$.

(i) Assume that $t<r, \tilde{r}$ and $q_{t+1}=\tilde{q}_{t+1}, p_{t+1}=\tilde{p}_{t+1}$. 


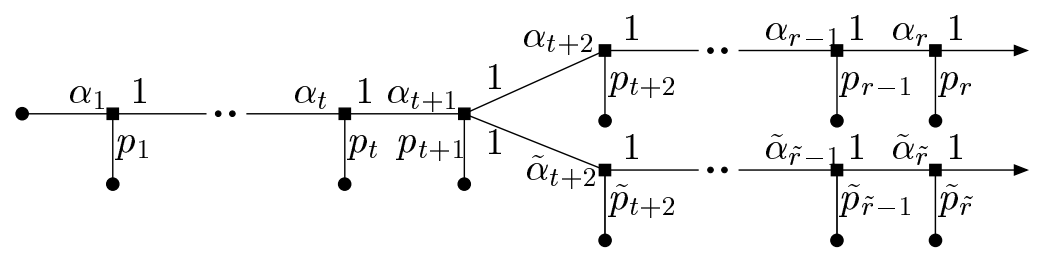

(ii) Assume that $t<r, \tilde{r}$ and $\frac{q_{t+1}}{p_{t+1}}<\frac{\tilde{q}_{t+1}}{\tilde{p}_{t+1}}$.

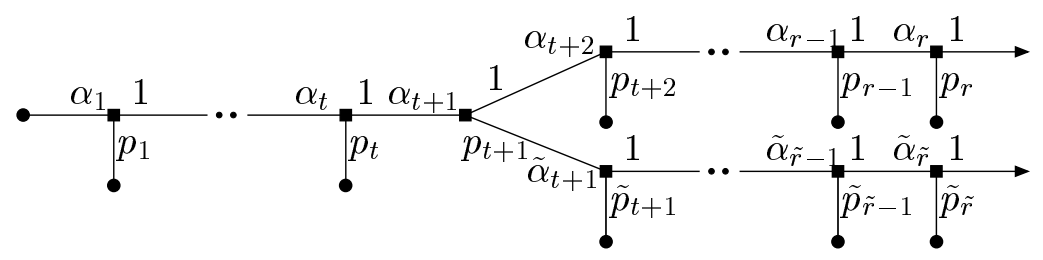

(iii) Assume that $t=\tilde{r}<r$. In this case, the diagram is obtained from the diagram in case (ii) by terminating the edge with weight $p_{t+1}$ by an arrowhead.

By interchanging $\Pi$ and $\tilde{\Pi}$ if necessary, (i-iii) define $\tilde{\Gamma}[f]$ in the case $\kappa=2$. The general case is obtained by induction on $\kappa$. The induction step involves operations of the kind (i-iii). Instead of giving the precise definition, we give a list of properties of the diagram $\tilde{\Gamma}=\tilde{\Gamma}[f]$ for an arbitrary singularity $[f]$. In the case $\kappa=1,2$, these properties are easily verified from the definitions above.

(A1) $\tilde{\Gamma}$ has the structure of a weighted tree. All weights are positive integers. (A2) $\tilde{\Gamma}$ has three kinds of vertices: arrowhead, knob and box vertices. The number of arrowhead vertices equals the number of branches of $[f]$. The arrowhead and knob vertices have 1 incoming edge, the boxed ones at least 3 . A box vertex has at most 2 neighboring knob vertices.

(A3) An edge of $\tilde{\Gamma}$ carries a weight at each ending box vertex. The edge-weights at a box vertex are pairwise relatively prime.

(A4) Let $b$ denote a box vertex of $\tilde{\Gamma}$. Let $E$ be the set of edges which connect $b$ to its neighboring box vertices. The graph $\tilde{\Gamma} \backslash E$ has $\# E+1$ connected components. There is at most one component which does neither contain $b$ nor any arrowhead vertex. Assume that there is such a component. Let $e$ be the edge which connects $b$ to that component and let $b^{\prime}$ denote the other vertex of $e$. Then $e$ has weight 1 at $b^{\prime}$.

(A5) Let $b, b^{\prime}$ denote connected box vertices of $\tilde{\Gamma}$. Let $a_{1}, \ldots, a_{k}$ be the weights at $b$ of the edges connecting $b$ and its neighboring vertices. 
Similarly, let $a_{1}^{\prime}, \ldots, a_{k^{\prime}}^{\prime}$ be the weights at $b^{\prime}$. Assume that $a_{1}, a_{1}^{\prime}$ are the weights of the edge connecting $b$ and $b^{\prime}$. Then

$$
a_{1} a_{1}^{\prime}-a_{2} \cdots a_{k} a_{2}^{\prime} \cdots a_{k^{\prime}}^{\prime}>0 \text {. }
$$

Step 4. The diagram $\Gamma=\Gamma[f]$ is obtained from $\tilde{\Gamma}=\tilde{\Gamma}[f]$ by the following algorithm. Set $\Gamma_{0}:=\tilde{\Gamma}$. STEP: Let $e$ be an edge of $\Gamma_{i}$ connecting a box vertex $b$ and a knob vertex $v$ and having weight 1 . If $e$ does not exist, set $\Gamma:=\Gamma_{i}$ and STOP. Otherwise, let $k \geq 3$ denote the number of incoming edges at $b$ and $n \leq 2$ the number of neighboring box vertices of $b$. If $k=3$ and $n=0$, set $\Gamma:=\Gamma_{*}:=\longleftrightarrow$ and STOP.

Otherwise, apply the operation $\Gamma_{i} \rightarrow \Gamma_{i+1}$ defined as follows

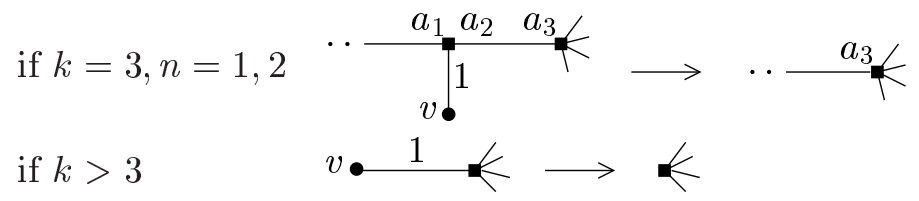

Finally repeat the STEP.

Among all the possible diagrams $\Gamma$ which are obtained by this algorithm, $\Gamma_{*}$ is exceptional in the sense that it does not have box vertices. It is important to note that in all other cases the properties (A1-5) still hold if $\tilde{\Gamma}$ is replaced by $\Gamma$. Additionally, we have

(A6) Every edge of $\Gamma$ connecting a box and a knob vertex has weight $>1$.

We end the discussion of splice diagrams with the remark that the diagram associated to the quadratic singularity $(x, y) \mapsto x^{2}+y^{2}$ is $\Gamma_{*}$.

B.2. Characteristic set. Let $\Gamma$ be the splice diagram of an isolated singularity. In this section, we define the set $\mathfrak{t}_{\Gamma} \subset \mathcal{T}$. In the exceptional case $\Gamma=\Gamma_{*}$, this is simply the set $\{(0,1,2 ; 1)\}$, which is the characteristic set of a positive Dehn twist. Assume from now on that $\Gamma \neq \Gamma_{*}$. Denote by $\mathcal{A}$ respectively $\mathcal{B}$ the set of arrowhead respectively box vertices of $\Gamma$. Moreover, denote by $\mathcal{E}$ the set of edges of $\Gamma$ which connect $\mathcal{B}$ to $\mathcal{A} \cup \mathcal{B}$.

In the following, we define (i) for each $b \in \mathcal{B}$ an element $t_{b} \in \mathcal{T}$ and (ii) for each $e \in \mathcal{E}$ an element $t_{e} \in \mathcal{T}$. We then set

$$
\mathfrak{t}_{\Gamma}:=\left\{t_{x}: x \in \mathcal{B} \cup \mathcal{E}\right\} .
$$

Finally we define (iii) for each $e \in \mathcal{E}$ an admissible twist map $\phi_{e}$.

Let $\mathcal{V}$ denote the set of ordered pairs of connected vertices of $\Gamma$. We start by introducing the function $m: \mathcal{V} \rightarrow \mathbb{N}$. Let $\left(v, v^{\prime}\right) \in \mathcal{V}$ and let $e$ 
be the edge connecting $v$ and $v^{\prime}$. The graph $\Gamma \backslash\{e\}$ has two components. Denote by $\Gamma^{\prime}$ that component which contains $v^{\prime}$. Let $\mathcal{A}^{\prime}$ denote the set of arrowhead vertices of $\Gamma^{\prime}$. For each $a \in \mathcal{A}^{\prime}$, there is a unique path in $\Gamma^{\prime}$, denoted by $\gamma_{a}$, which connects $v^{\prime}$ and $a$. Define $\sigma_{a}>0$ to be the product of all edge-weights adjacent to $\gamma_{a}$, but not on $\gamma_{a}$. For examples see [10, page 84]. If $\gamma_{a}$ is the constant path, set $\sigma_{a}:=1$. Define

$$
m\left(v, v^{\prime}\right):=\sum_{a \in \mathcal{A}^{\prime}} \sigma_{a}
$$

Note that $m\left(v, v^{\prime}\right)=0$ if and only if $\mathcal{A}^{\prime}=\emptyset$. Together with (A4), this has the following consequences:

(B1) If $m\left(v, v^{\prime}\right)=0$, then $m\left(v^{\prime}, v\right) \neq 0$.

(B2) If $b, b^{\prime} \in \mathcal{B}$ and $m\left(b, b^{\prime}\right)=0$, then the edge connecting $b$ and $b^{\prime}$ has weight 1 at $b^{\prime}$.

(B3) For each $b \in \mathcal{B}$, there exists at most one neighboring vertex $b^{\prime} \in \mathcal{B}$ such that $m\left(b, b^{\prime}\right)=0$.

(B4) If $b \in \mathcal{B}$ and $a \in \mathcal{A}$, then $m(b, a)=1$.

(i) Let $b \in \mathcal{B}$ and denote by $\left\{v_{1}, \ldots, v_{n}, v_{n+1}, \ldots, v_{k}\right\}, 1 \leq n \leq k$, the set of vertices which are connected to $b$, ordered such that $v_{i} \in \mathcal{A} \cup \mathcal{B}$ if and only if $i \leq n$. Note that $k \geq 3$ and that $k-n \in\{0,1,2\}$. Further denote by $a_{i}>0, i=1, \ldots, k$, the weight at $b$ of the edge connecting $b$ and $v_{i}$. Define the numbers

$$
d_{b}:=\operatorname{gcd}\left(m\left(b, v_{1}\right), \ldots, m\left(b, v_{n}\right)\right), \quad h_{b}:=\sum_{i=1}^{n} \operatorname{gcd}\left(m\left(b, v_{i}\right), m\left(v_{i}, b\right)\right)
$$

and

$$
\ell_{b}:=\sum_{i=1}^{n} m\left(b, v_{i}\right) \cdot a_{1} \cdots \widehat{a}_{i} \cdots a_{k}, \quad \chi_{b}:=\ell_{b} \cdot\left(2-k+\sum_{i=n+1}^{k} \frac{1}{a_{i}}\right),
$$

where the hat means that the underlying factor is omitted. Note that (B3), (B4) respectively (B1) imply that $d_{b}$ respectively $h_{b}$ is well defined and therefore $>0$. Similarly, it follows from (B3), (B4) that the integer $\ell_{b}$ is $>0$. We furthermore claim that $\chi_{b}<0$. This is because $k \geq 3$ and $a_{n+1}, \ldots, a_{k}>1$ are pairwise relatively prime. Hence, we can set

$$
t_{b}:=\left(\chi_{b}, d_{b}, h_{b} ; \ell_{b}\right) .
$$

Finally note that from the definition of $m$, it follows that for each $i=1, \ldots, n$,

$$
\ell_{b}=m\left(b, v_{i}\right) \cdot a_{1} \cdots \widehat{a_{i}} \cdots a_{k}+m\left(v_{i}, b\right) \cdot a_{i} .
$$


(ii) Let $e \in \mathcal{E}$ be an edge which connects the vertices $b, b^{\prime} \in \mathcal{B}$. Let $a_{1}, \ldots, a_{k}$ be the weights at $b$ of the edges connecting $b$ and its neighboring vertices. Similarly, let $a_{1}^{\prime}, \ldots, a_{k^{\prime}}^{\prime}$ be the weights at $b^{\prime}$. Assume that $a_{1}, a_{1}^{\prime}$ are the weights of $e$. Define the numbers

$$
d_{e}:=\operatorname{gcd}\left(m\left(b, b^{\prime}\right), m\left(b^{\prime}, b\right)\right), \quad \Delta_{e}:=a_{1} a_{1}^{\prime}-a_{2} \cdots a_{k} a_{2}^{\prime} \cdots a_{k^{\prime}}^{\prime}
$$

and

$$
\ell_{e}:=\frac{d_{e} \cdot \Delta_{e}}{\ell_{b} \cdot \ell_{b^{\prime}}}
$$

By $(\mathrm{B} 1), d_{e}$ is well defined and therefore $>0$. Set

$$
t_{e}:=\left(0, d_{e}, 2 d_{e} ; \ell_{e}\right) \in \mathcal{T} \text {. }
$$

Now let $e \in \mathcal{E}$ be an edge which connects the vertex $b \in \mathcal{B}$ to an arrowhead vertex. In this case set

$$
t_{e}:=\left(0, d_{e}, 2 d_{e} ; \ell_{e}\right):=\left(0,1,2 ; 1 / \ell_{b}\right) \in \mathcal{T} .
$$

(iii) Let $e$ be an edge which connects $b \in \mathcal{B}$ and $b^{\prime} \in \mathcal{A} \cup \mathcal{B}$. By (B1),(B4) we can assume without loss of generality that $m\left(b, b^{\prime}\right) \neq 0$. Now choose integers $n, n^{\prime}$ with $m\left(b, b^{\prime}\right) \cdot n^{\prime}+m\left(b^{\prime}, b\right) \cdot n=d_{e}$. Denote by $a$ the weight of $e$ at $b$ and set $m:=m\left(b, b^{\prime}\right)$. Define $\phi_{e}$ to be the admissible twist map which cyclically permutes $d_{e}$ annuli and such that

$$
\phi_{e}^{d_{e}}(q, p)=\left(q, p-q \cdot d_{e} \ell_{e}-\frac{d_{e}}{m}\left(n-\frac{d_{e} \cdot a}{\ell_{b}}\right)\right),
$$

for all $(q, p) \in[0,1] \times S^{1}$.

B.3. Proof of Proposition 19. We first state the precise results that are needed for the proof.

Theorem 23 (Eisenbud, Neumann). Let $[f]$ be an isolated plane curve singularity with Milnor fiber $M$ and geometric monodromy $g$. There exists an admissible triple $(\phi, M, C)$ such that $\phi \in \iota_{*} g$ and $\mathfrak{t}(\phi, M, C)=$ $\mathfrak{t}_{\Gamma[f]}$. Moreover, the twist map components of $\phi$ are given by the model (19).

Remarks. (i) This theorem is a summary of results from Sections 9, 10, 11 and 13 of [10]. The periodic components of the monodromy are described in Lemma 11.4 and the twist map components in Theorems 13.1, 13.5. The positive twist property (A5) is contained in Theorem 9.4. We remark that our notation differs at some points from that of $[\mathbf{1 0}]$.

(ii) Eisenbud, Neumann give a detailed description of the periodic components of the monodromy in terms of cyclic branched coverings in $[\mathbf{1 0}$, Lemma 11.4]. 
(iii) The graph $\Gamma[f]$ contains more information than the characteristic set $\mathfrak{t}_{\Gamma[f]}$. It also shows how the $\phi$-components are pieced together to give the Milnor fiber.

The second main ingredient for our proof is the following result of [2] and [17]. We would like to point out that this result holds in much greater generality than we use it here, namely for holomorphic hypersurface singularities, isolated or non-isolated, in any dimension.

Theorem 24 (A'Campo, Lê). Let $g$ be the geometric monodromy of an isolated plane curve singularity. Then $\Lambda(g)=0$, where $\Lambda$ denotes the Lefschetz number.

Proof of Proposition 19. Let $[f]$ be an isolated plane curve singularity. Let $M$ denote the Milnor fiber and $g$ the geometric monodromy of $[f]$. By Theorem 23, there exists a representative $\phi \in \iota_{*} g$ and a finite $\phi$ invariant union of circles $C \subset M$, such that if $M^{\prime}$ is a $\phi$-component of $M \backslash C$, then either $M^{\prime}$ has negative Euler characteristic and $\phi \mid M^{\prime}$ is periodic, or $M^{\prime}$ is a union of annuli and $\phi \mid M^{\prime}$ is an admissible twist map. If $(\chi, d, h)$ denotes the topological type of $M^{\prime}$ and $\ell$ the order/twist number of $\phi \mid M^{\prime}$, then $(d, \chi, h ; \ell) \in \mathfrak{t}_{\Gamma[f]}$. Moreover, if $M^{\prime}$ is a union of annuli, then (19) is a model for $\phi \mid M^{\prime}$.

The strategy of the proof is now as follows. We will prove below that

Claim 1. If $\chi=0$, then $\operatorname{Fix}(\phi) \cap \operatorname{int}\left(M^{\prime}\right)=\emptyset$.

Claim 2. If $\chi<0$, then $\ell>1$.

Claim 3. $\phi$ only has positive twists.

Claim 1 implies that $\phi$ is a diffeomorphism of finite type. From Proposition 9 about the fixed point classes of a diffeomorphism of finite type and claim 2, it follows that $\operatorname{Fix}(\phi) \cap \operatorname{int}(M)$ is a discrete set of fixed points with fixed point index 1. The Lefschetz fixed point theorem therefore implies that

$$
\Lambda(\phi)=\#(\operatorname{Fix}(\phi) \cap \operatorname{int}(M)) .
$$

Note that $\partial M$ has fixed point index 0 . From Theorem 24, it hence follows that $\operatorname{Fix}(\phi) \cap \operatorname{int}(M)=\emptyset$. Together with claim 3, this proves Proposition 19, up to claim 1,2 and 3.

Note that if $\Gamma[f]=\Gamma_{*}$, then $M$ is an annulus and $\phi$ is a positive Dehn twist. Claim 1,2 and 3 obviously hold in this case and we assume from now on that $\Gamma[f] \neq \Gamma_{*}$. We begin by proving claim 2 . Recall that $\mathfrak{t}_{\Gamma[f]}=\left\{t_{x}: x \in \mathcal{B} \cup \mathcal{E}\right\}$ and that if $\chi<0$, there exists $b \in \mathcal{B}$ such 
that $(\chi, d, h ; \ell)=\left(\chi_{b}, d_{b}, h_{b} ; \ell_{b}\right)$. Consider equation (16) and assume that $\ell_{b}=1$. In this case, only one summand of $\ell_{b}$ is non-zero, which, by (B3), is only possible if $k \leq 2$. Since $n \geq 3$, however, it follows from (A6) that $a_{n}>1$ and hence that $1=\ell_{b} \geq a_{n}>1$, a contradiction.

To proof claim 1 , let $e \in \mathcal{E}$ be such that $(\chi, d, h ; \ell)=\left(0, d_{e}, 2 d_{e} ; \ell_{e}\right)$. We can assume that $d_{e}=1$, otherwise the claim is obviously true. Hence $M^{\prime}$ is an annulus and $\phi \mid M^{\prime}=\phi_{e}$. Consider equation (19) and assume that $\phi_{e}(q, p)=(q, p)$ for some $(q, p) \in(0,1) \times S^{1}$. This is only possible if

$$
-q \cdot \ell_{e}-\frac{1}{m}\left(n-\frac{a}{\ell_{b}}\right) \in \mathbb{Z}
$$

where we use the same notation as in (19). This in turn implies that

$$
a-q m \ell_{b} \ell_{e} \in \ell_{b} \mathbb{Z} .
$$

Assume for the moment that $0<m \ell_{b} \ell_{e} \leq a$. Since $0<q<1$, it follows from (20) that $0<\ell_{b}<a$. To show that this is a contradiction, recall from (17) that $\ell_{b}=m a_{2} \cdots a_{r}+m^{\prime} a$, where $m^{\prime}:=m\left(b^{\prime}, b\right)$. If $m^{\prime} \neq 0$, it follows that $\ell_{b} \geq a$ and hence $m^{\prime}=0$. By (B2) however, this implies that $a=1>\ell_{b}$, which is a contradiction.

It remains to prove that $0<m \ell_{b} \ell_{e} \leq a$. First note that $m \ell_{b} \ell_{e}>0$ iff $\ell_{e}>0$. If $b^{\prime} \in \mathcal{A}$, then $\ell_{b}>0$ by definition. If $b \in \mathcal{B}$, then (A5) is exactly the statement that $\ell_{e}>0$. This in fact proves claim 3 . To prove that $m \ell_{b} \ell_{e} \leq a$, first assume that $b^{\prime} \in \mathcal{A}$. Then $m=1, a=1, \ell_{b} \ell_{e}=1$ and we are finished. If $b^{\prime} \in \mathcal{B}$, then it follows from (17) and (18) that

$$
\ell_{b^{\prime}} \geq m a^{\prime}, \quad \Delta_{e} \leq a a^{\prime},
$$

where $a^{\prime}$ denotes the weight of $e$ at $b^{\prime}$. This implies that

$$
\ell_{e} \leq \frac{a}{\ell_{b} m}
$$

which proves the required inequality. This ends the proof of the proposition.

\section{Appendix C. Floer homology on surfaces with boundary}

Let $M$ be a compact connected oriented 2-manifold with boundary $\partial M \neq \emptyset$. Recall that $\operatorname{Diff}_{c}^{+}(M)$ denotes the group of orientation preserving diffeomorphisms which are the identity near $\partial M$. This appendix addresses Floer homology theory for elements of $\operatorname{Diff}_{c}^{+}(M)$. In higher dimensions, this is known as Floer homology theory for exact symplectomorphisms of exact symplectic manifolds with contact type boundary and was used in [27, Section 4], see also [7]. Due to the dimensional 
restriction, the theory exhibits auxiliary structure, namely isotopy invariance, as in the closed case. The central notion around this issue is that of monotonicity. We start by defining monotonicity and show that it has naturality, isotopy and inclusion properties similar to the ones discussed on page 720 for the closed case.

Let $\omega$ be an area form on $M$ and denote by $\operatorname{Symp}_{c}(M, \omega)$ the group of $\omega$-preserving diffeomorphisms which are the identity near $\partial M$. If $\phi \in \operatorname{Symp}_{c}(M, \omega), \omega$ induces a closed 2-form $\omega_{\phi}$ on the mapping torus $T_{\phi}$.

Definition 25. $\phi \in \operatorname{Symp}_{c}(M, \omega)$ is called monotone if

$$
\left[\omega_{\phi}\right]=0
$$

in $H^{2}\left(T_{\phi} ; \mathbb{R}\right)$. $\operatorname{Symp}_{c}^{m}(\Sigma, \omega)$ denotes the set of monotone symplectomorphisms.

As in the closed case it is useful to look at the short exact sequence

$$
0 \longrightarrow \frac{H^{1}(M ; \mathbb{R})}{\operatorname{im}\left(\mathrm{id}-\phi^{*}\right)} \stackrel{\delta}{\longrightarrow} H^{2}\left(T_{\phi} ; \mathbb{R}\right) \stackrel{\iota^{*}}{\longrightarrow} H^{2}(M ; \mathbb{R})=0 \longrightarrow 0
$$

and define the class $m(\phi) \in H^{1}(M ; \mathbb{R}) / \mathrm{im}\left(\mathrm{id}-\phi^{*}\right)$ satisfying $\delta m(\phi)=$ $\left[\omega_{\phi}\right]$. The naturality, isotopy and inclusion properties discussed on page 720 in the closed case carry over word by word to the current situation with the addition of a subscript $c$ to all diffeomorphism groups. For the first two properties this is straight forward to check. The inclusion property needs separate consideration. Recall:

(Inclusion) The inclusion $\operatorname{Symp}_{c}^{m}(M, \omega) \hookrightarrow \operatorname{Diff}_{c}^{+}(M)$ is a homotopy equivalence. In particular, every connected component of $\operatorname{Symp}_{c}^{m}(\Sigma, \omega)$ is contractible.

The proof is analogous to the closed case and uses the following three facts. Firstly, the inclusion $\operatorname{Symp}_{c}(M, \omega) \hookrightarrow \operatorname{Diff}_{c}^{+}(M)$ is a homotopy equivalence. This follows from an extension of Moser's theorem, see [19, Exercise 3.18]. Secondly, every connected component of $\operatorname{Diff}_{c}^{+}(M)$ is contractible. If the genus of $M$ is $\neq 0$, this is shown using the EarlEells Theorem [8]. If $g=0$, it follows from the corresponding result for the disk, which is due to Smale [29, Theorem B]. Thirdly, we have

Lemma 26. If $\phi \in \operatorname{Symp}_{c}(M, \omega)$, there exists a closed 1-form $\theta \in m(\phi)$ such that $\operatorname{supp}(\theta) \subset \operatorname{int}(M)$. The flow $\left(\psi_{t}\right)_{t \in \mathbb{R}}$ of the vector field $X$ which is uniquely defined by $\omega(X, \cdot)=-\theta$, satisfies $\psi_{t} \in \operatorname{Symp}_{c}(M, \omega)$ and $m\left(\phi \circ \psi_{1}\right)=0$. 
Proof. The second part of the statement follows immediately from the first one and the isotopy property:

$$
m\left(\phi \circ \psi_{1}\right)=m(\phi)+\left[\operatorname{Flux}\left(\psi_{t}\right)\right]
$$

in $H^{1}(M ; \mathbb{R}) / \mathrm{im}\left(\mathrm{id}-\phi^{*}\right)$. To prove the first statement, let $\beta \in m(\phi)$ be a closed 1-form. Let $S_{1}, \ldots, S_{n}$ denote the connected components of $\partial M$ and choose for each $i$ a collar neighborhood $N_{i} \subset M$ of $S_{i}$ and a closed 1 -form $\theta_{i}$ on $N_{i}$ such that $\left\langle\left[\theta_{i}\right],\left[S_{i}\right]\right\rangle=1$. There exist $f: M \rightarrow \mathbb{R}$ smooth and $t_{1}, \ldots, t_{n} \in \mathbb{R}$ such that

$$
(\beta+\mathrm{d} f) \mid N_{i}=t_{i} \cdot \theta_{i}, \quad \forall i=1, \ldots, n .
$$

We claim that $\theta:=\beta+\mathrm{d} f$ is the required 1-form. Recall from page 719 that $\delta: H^{1}(M ; \mathbb{R}) \rightarrow H^{2}\left(T_{\phi} ; \mathbb{R}\right)$ is given by $\delta[\theta]=[\rho \cdot \theta \wedge \mathrm{d} t]$, with $\rho:[0,1] \rightarrow \mathbb{R}$ a smooth function vanishing near 0 and 1 , and satisfying $\int_{0}^{1} \rho \mathrm{d} t=1$. Furthermore note that $S^{1} \times S_{i} \subset T_{\phi}$ is an embedded 2-torus for each $i=1, \ldots, n$. Using (21), it follows that

$$
\begin{aligned}
\left\langle\left[\omega_{\phi}\right],\left[S^{1} \times S_{i}\right]\right\rangle & =\left\langle\delta[\alpha],\left[S^{1} \times S_{i}\right]\right\rangle \\
& =-\left\langle[\rho \cdot \mathrm{d} t],\left[S^{1}\right]\right\rangle \cdot\left\langle[\alpha],\left[S_{i}\right]\right\rangle \\
& =-t_{i} \cdot\left\langle\left[\theta_{i}\right],\left[S_{i}\right]\right\rangle=-t_{i} .
\end{aligned}
$$

On the other hand,

$$
\left\langle\left[\omega_{\phi}\right],\left[S^{1} \times S_{i}\right]\right\rangle=0,
$$

since $\omega_{\phi}$ has no $\mathrm{d} t$-component. Hence, $t_{i}=0$ for all $i=1, \ldots, n$, which proves the claim.

Recall that in the closed case monotonicity firstly guarantees compactness of the space of Floer connecting orbits and secondly is used to prove invariance. The same holds in the current situation.

(Floer homology) To every $\phi \in \operatorname{Symp}_{c}^{m}(\Sigma, \omega)$ symplectic Floer homology theory assigns a pair of $\mathbb{Z}_{2}$-graded vector spaces $H F_{*}(\phi, \pm)$ over $\mathbb{Z}_{2}$, with multiplicative structures

$$
H^{*}\left(M ; \mathbb{Z}_{2}\right) \otimes H F_{*}(\phi, \pm) \longrightarrow H F_{*}(\phi, \pm) .
$$

Floer homology is natural in the sense that $H F_{*}(\phi, \pm)$ and $H F_{*}\left(\psi^{*} \phi, \pm\right)$ are naturally isomorphic as modules over $H^{*}\left(M ; \mathbb{Z}_{2}\right)$, for all $\psi \in \operatorname{Diff}_{c}^{+}(M)$.

(Invariance) If $\phi$ and $\phi^{\prime} \in \operatorname{Symp}_{c}^{m}(\Sigma, \omega)$ are isotopic in $\operatorname{Diff}_{c}^{+}(M)$, then $H F_{*}(\phi, \pm)$ and $H F_{*}\left(\phi^{\prime}, \pm\right)$ are naturally isomorphic as $H^{*}\left(M ; \mathbb{Z}_{2}\right)$ modules. The appearance of the sign in the Floer homology corresponds 
to two ways of perturbing $\phi \in \operatorname{Symp}_{c}^{m}(\Sigma, \omega)$ near $\partial M$. To be more precise, let $\jmath: \bigcup(-\varepsilon, 0] \times S^{1} \rightarrow M$ be a collar neighborhood of $\partial M$, such that $\jmath^{*} \omega=\mathrm{d} q \wedge \mathrm{d} p$ with $(q, p) \in(-\varepsilon, 0] \times S^{1}$. Choose $H: M \rightarrow \mathbb{R}$ with support near $\partial M$ and such that $\jmath^{*} H(q, p)=-q$. Let $\psi_{t}$ denote the Hamiltonian flow generated by $H$, choose $0<\delta<1$ and set

$$
\phi_{+}:=\phi \circ \psi_{\delta}, \quad \phi_{-}:=\phi \circ \psi_{-\delta} .
$$

The definition of the Floer complex for $\phi_{ \pm}$is along the same line as that in the closed case [28], with the usual modifications that are needed in the presence of a contact type boundary.

The modifications include a condition on the path $J=\left(J_{t}\right)_{t \in \mathbb{R}}$ of $\omega$-compatible complex structures that is used to define the Floer connecting orbits; namely that $\jmath^{*} J_{t}$ is the standard complex structure on $\bigcup(-\varepsilon, 0] \times S^{1}$, for all $t \in \mathbb{R}$. We briefly recall the use of this condition. Assume without loss of generality that $\phi \mid$ im $\jmath=$ id. Now let $u: \mathbb{R}^{2} \rightarrow M$ be a smooth map satisfying

$$
\left\{\begin{array}{l}
u(s, t)=\phi_{+}(u(s, t+1)), \\
\partial_{s} u+J_{t}(u) \partial_{t} u=0, \\
\lim _{s \rightarrow \pm \infty} u(s, t) \in \operatorname{Fix}\left(\phi_{+}\right) .
\end{array}\right.
$$

We claim that $\operatorname{im} u \subset M \backslash \operatorname{im} \jmath$. Assume by contradiction that $u^{-1}(\operatorname{im} \jmath)$ is non-empty and let $u_{q}: u^{-1}(\operatorname{im} \jmath) \rightarrow \mathbb{R}$ denote the $q$-component of $\jmath^{-1} \circ u$. By construction, $u_{q}$ is smooth and not locally constant. Using the first and third equation in (22), one can now show that $u_{q}$ has a global maximum. From the second equation in (22) together with the above assumption on $J_{t}$, it furthermore follows that $u_{q}$ is a harmonic function. This contradicts the maximum principle and hence proves the claim, which assures that the Floer connecting orbits are contained in a compact subset of $\operatorname{int}(M)$.

We close this section by remarking that $H F_{*}(\phi,+), H F_{*}(\phi,-)$ are independent of the choices of the local chart $\}$ and perturbation data $H, \delta$. They are invariants of the isotopy class of $\phi$ in $\operatorname{Diff}_{c}^{+}(M)$. 


\section{Bibliography}

[1] N. A'Campo. La fonction zêta d'une monodromy. Comment. Math. Helvetici, 50:233-248, 1975.

[2] N. A'Campo. On monodromy maps of hypersurface singularities. In Manifolds-Tokyo 1973, pages 151-152. University of Tokyo Press, 1973.

[3] N. A'Campo. Real deformations and complex topology of plane curve singularities. Ann. Fac. Sci. Toulouse Math., 8(1):5-23, 1999.

[4] N. A'Campo. Sur la monodromie des singularités isolée d'hypersurface complexes. Invent. Math., 20:147-170, 1973.

[5] V. I. Arnold, S. M. Gusein-Zade, and A. N. Varchenko. Singularities of differentiable maps, volume II, volume 83 of Monographs in Mathematics. Birckhäuser, 1988.

[6] E. Brieskorn and H. Knörrer. Plane algebraic curves. Birckhäuser, 1986.

[7] K. Cieliebak, A. Floer, and H. Hofer. Symplectic homology II. A general construction. Math. Z., 218(1):103-122, 1995.

[8] C. J. Earle and J. Eells. The diffeomorphism group of a compact Riemann surface. Bull. Amer. Math. Soc., 73:557-559, 1967.

[9] E. Eftekhary. Floer cohomology of certain pseudo-Anosov maps on surfaces. Preprint, 2002. math.SG/0205029.

[10] D. Eisenbud and W. Neumann. Three-dimensional link theory and invariants of plane curve singularities, volume 110 of Annals of Mathematical Studies. Princeton University Press, 1985.

[11] A. Floer. Symplectic fixed points and holomorphic spheres. Comm. Math. Phys., 120(2):575-611, 1989.

[12] D. Fried. Monodromy and dynamical systems. Topology, 25(4):443-453, 1986.

[13] R. Gautschi. Freiheitssatz for surface groups. Preprint, April 2002.

[14] M. Hutchings and M. Sullivan. The periodic Floer homology of a 
Dehn twist. Preprint, 2002.

[15] B. Jiang. Fixed point classes from a differentiable viewpoint. In Fixed point theory, volume 886 of Lecture Notes in Math., pages 163-170. Springer, 1981.

[16] B. Jiang and J. Guo. Fixed points of surface diffeomorphisms. Pac. J. Math, 160(1):67-89, 1993.

[17] D. T. Lê. La monodromie n'a pas de points fixes. J. Fac. Sci. Univ. Tokyo Sect. IA Math., 22(3):409-427, 1975.

[18] J. Milnor. Singular points of complex hypersurfaces, volume 61 of Annals of Mathematical Studies. Princeton University Press, 1968.

[19] D. McDuff and D. A. Salamon. Introduction to symplectic topology. Oxford Mathematical Monographs. Oxford Science Publications, 1998.

[20] J. Moser. On the volume elements on a manifold. Trans. Amer. Math. Soc., 120:286-294, 1965.

[21] W. Neumann. Splicing algebraic links. In Complex analytic singularities, volume 8 of Adv. Stud. Pure Math., pages 349-361. North Holland, 1987.

[22] J. Nielsen. Surface transformations of algebraically finite type. Danske Vid. Salsk. Math.-Phys., 21, 1944.

[23] D. A. Salamon and E. Zehnder. Morse theory for solutions of Hamiltonian systems and the Maslov index. Comm. Pure Appl. Math., 45(10):1303-1360, 1992.

[24] M. Schwarz. Morse homology, volume 111 of Progress in Mathematics. Birckhäuser, 1993.

[25] M. Poźniak. Floer homology, Novikov rings and clean intersections. In Northern California Symplectic Geometry Seminar, volume 196 of Amer. Math. Soc. Transl. Ser.2, pages 119-181. American Mathematical Society, 1999.

[26] P. Seidel. Floer homology of a Dehn twist. Math. Res. Lett., 3(6):829-834, 1997.

[27] P. Seidel. More on vanishing cycles and mutation. Preprint, October 2000. math.SG/0010032.

[28] P. Seidel. Symplectic Floer homology and the mapping class group. Preprint, March 2001. math.SG/0010301.

[29] S. Smale. Diffeomorphisms of the 2-sphere. Proc. Amer. Math. Soc., 10:621-626, 1959.

[30] W. P. Thurston. On the geometry and dynamics of diffeomorphisms of surfaces. Bull. Amer. Math. Soc., 19(2):417-431, October 1988 . 
ETH ZÜRICH

Current address:

Bergstrasse 76, 8032 Zürich

E-mail address: ralf_gautschi@yahoo.de

Research partially supported by the SNF (project number 21-64937.01). 